\title{
Nonvarying sums of Lyapunov exponents of Abelian differentials in low genus
}

\author{
DAWEI CHEN \\ MARTIN MÖLLER
}

\begin{abstract}
We show that for many strata of Abelian differentials in low genus the sum of Lyapunov exponents for the Teichmüller geodesic flow is the same for all Teichmüller curves in that stratum, hence equal to the sum of Lyapunov exponents for the whole stratum. This behavior is due to the disjointness property of Teichmüller curves with various geometrically defined divisors on moduli spaces of curves.
\end{abstract}

14H10; 37D40, 14H51

\section{Introduction}

Lyapunov exponents of dynamical systems are often hard to calculate explicitly. For the Teichmüller geodesic flow on the moduli space of Abelian differentials at least the sum of the positive Lyapunov exponents is accessible for two cases. The moduli space decomposes into various strata, each of which carries a finite invariant measure with full support. For these measures the sum of Lyapunov exponents can be calculated using results of Eskin, Masur and Zorich [16] together with results of Eskin and McMullen [15]. On the other hand, the strata contain many Teichmüller curves, eg those generated by square-tiled surfaces. For Teichmüller curves an algorithm in [15] calculates the sum of Lyapunov exponents, of course only one Teichmüller curve at a time.

On several occasions, one likes to have estimates, or even the precise values of Lyapunov exponents for all Teichmüller curves in the same stratum simultaneously. For example, it is shown by Delecroix, Hubert and Lelièvre in [13] that Lyapunov exponents are responsible for the rate of diffusion in the wind-tree model, where the parameters of the obstacle correspond to picking a flat surface in a fixed stratum. One would like to know this escape rate not only for a specific choice of parameters nor for the generic value of parameters but for all parameters.

Zorich communicated to the authors, that, based on a limited number of computer experiments about a decade ago, Kontsevich and Zorich observed that the sum of 
Lyapunov exponents is nonvarying among all the Teichmüller curves in a stratum roughly if the genus plus the number of zeros is less than seven, while the sum varies if this sum is greater than seven.

In this paper we show that a more precise version of this numerical observation indeed is true. More precisely, we treat the moduli space of genera less than or equal to five. For each of its strata - with three spin-related exceptions - we either exhibit an example showing that the sum is varying — the easy part—or prove that the sum is nonvarying. The latter will be achieved by showing empty intersection of Teichmüller curves with various geometrically defined divisors on moduli spaces of curves. We remark that each stratum requires its own choice of divisor and its individual proof of disjointness, with varying complexity of the argument. In complement to our low genus results we mention a theorem of [15] that shows that for all hyperelliptic loci the sum of Lyapunov exponents is nonvarying.

We now give the precise statement of what emerged out of the observation by Kontsevich and Zorich. Let $\left(m_{1}, \ldots, m_{k}\right)$ be a partition of $2 g-2$. Denote by $\Omega \mathcal{M}_{g}\left(m_{1}, \ldots, m_{k}\right)$ the stratum parameterizing genus $g$ Riemann surfaces with Abelian differentials that have $k$ distinct zeros of order $m_{1}, \ldots, m_{k}$. We say that the sum of Lyapunov exponents is nonvarying in (a connected component of) a stratum $\Omega \mathcal{M}_{g}\left(m_{1}, \ldots, m_{k}\right)$, if for all Teichmüller curves generated by a flat surface in $\Omega \mathcal{M}_{g}\left(m_{1}, \ldots, m_{k}\right)$ its sum of Lyapunov exponents equals the sum for the finite invariant measure supported on (the area one hypersurface of) the whole stratum.

Theorem 1.1 For all strata in genus $g=3$ but the principal stratum the sum of Lyapunov exponents is nonvarying.

For the principal stratum, the sum of Lyapunov exponents is bounded above by 2 . This bound can be attained for Teichmüller curves in the hyperelliptic locus, eg, for Teichmüller curves that are unramified double covers of genus two curves and also for Teichmüller curves that do not lie in the hyperelliptic locus.

Theorem 1.2 For the strata with signature $(6)^{\text {even }},(6)^{\text {odd }},(5,1),(3,3),(3,2,1)$ and $(2,2,2)^{\text {odd }}$ as well as for the hyperelliptic strata in genus $g=4$ the sum of Lyapunov exponents is nonvarying.

For all the remaining strata, except maybe $(4,2)^{\text {odd }}$ and $(4,2)^{\text {even }}$, the sum of Lyapunov exponents is varying and bounded above by $5 / 2$.

We give more precise upper bounds for the sum stratum by stratum in the text. We remark that eg for $\Omega \mathcal{M}_{4}(4,1,1)$ the sharp upper bound is $23 / 10$, which is attained for 
hyperelliptic curves, whereas for all nonhyperelliptic curves in this stratum the sum of Lyapunov exponents is bounded above by $21 / 10$. This special role of the hyperelliptic locus is visible throughout the paper.

For $g=5$, since there are quite a lot of strata, we will not give a full discussion of upper bounds for varying sums, but restrict to the cases where the sum is nonvarying.

Theorem 1.3 For the strata with signature $(8)^{\text {even }},(8)^{\text {odd }}$ and $(5,3)$ as well as for the hyperelliptic strata in genus $g=5$ the sum of Lyapunov exponents is nonvarying.

For all the other strata, except maybe $(6,2)^{\text {odd }}$, the sum of Lyapunov exponents is varying.

We also expect the three unconfirmed cases $(4,2)^{\text {even }},(4,2)^{\text {odd }}$ and $(6,2)^{\text {odd }}$ to be nonvarying ${ }^{1}$, but a proof most likely requires a good understanding of the moduli space of spin curves, on which much less is known than on the moduli space of curves.

The above theorems seem to be the end of this nonvarying phenomenon. We cannot claim that there is not a single further stratum of genus greater than five and not hyperelliptic, where the sum is nonvarying. But while the sum in strata with a single zero is always nonvarying for $g \leq 5$, the sum does vary in both nonhyperelliptic components of the stratum $\Omega \mathcal{M}_{6}(10)$, as we show in Proposition 7.4.

As mentioned above, by [15] for hyperelliptic strata in any genus the sum of Lyapunov exponents is nonvarying. This has significance not only in dynamics, but also in the study of birational geometry of moduli spaces. In Theorem 8.1 we mention one application to the extremality of certain divisor classes on the moduli space of pointed curves, which answers a question posed by Harris [25, page 413] and Harris and Morrison [27, Problem (6.34)].

We now describe our strategy. One can associate the three quantities of 'slope', 'SiegelVeech constant' and 'the sum of Lyapunov exponents' to a Teichmüller curve. Any one of the three determines the other two. Hence it suffices to verify the nonvarying property for slopes. To do this, we exhibit a geometrically defined divisor on the moduli space of curves and show that Teichmüller curves in a stratum do not intersect this divisor. It implies that those Teichmüller curves have the same slope as that of the divisor.

The slope of the divisors, more generally their divisor classes in the Picard group of the moduli space, can be retrieved from the literature in most cases we need. In the

\footnotetext{
${ }^{1}$ Recently Yu and Zuo [41] confirmed the three cases using filtration of the Hodge bundle. See also the authors' [9, Theorem A.9] for a detailed explanation.
} 
remaining cases, we apply the standard procedure using test curves to calculate the divisor class.

Frequently, we also need to consider the moduli space of curves with marked points or spin structures, but the basic idea remains the same. For upper bounds of sums of Lyapunov exponents, they follow from the nonnegative intersection property of Teichmüller curves with various divisors on moduli spaces.

Technically, some of the complications arise from the fact that the disjointness of a Teichmüller curve with a divisor is relatively easy to check in the interior of the moduli space, but requires extra care when dealing with stable nodal curves in the boundary.

In a sequel paper [9] we consider Teichmüller curves generated by quadratic differentials and verify many nonvarying strata of quadratic differentials in low genus. These results immediately trigger a number of questions. Just to mention the most obvious ones: What about measures supported on manifolds of intermediate dimension? What about the value distribution for the sums in a stratum where the sum is varying? We hope to treat these questions in the future.

This paper is organized as follows. In Sections 2, 3 and 4 we give a background introduction to moduli spaces and their divisors, as well as to Teichmüller curves and Lyapunov exponents. In particular, in Section 3.3 we study the properties of Teichmüller curves that are needed in the proof and in Section 4.3 we describe the upshot of our strategy. Our main results for $g=3, g=4$ and $g=5$ are proved in Sections 5, 6 and 7 , respectively. Finally in Section 8 we discuss an application of the Teichmüller curves in the hyperelliptic strata to the geometry of moduli spaces of pointed curves.

Acknowledgements This work was initiated when the first author visited the Hausdorff Research Institute for Mathematics in Summer 2010. Both authors thank HIM for hospitality. The evaluation of Lyapunov exponents were performed with the help of computer programs of Anton Zorich and Vincent Delecroix. The authors are grateful to Anton and Vincent for sharing the programs and the data. The first author also would like to thank Izzet Coskun for stimulating discussions on the geometry of canonical curves and thank Alex Eskin for leading him into the beautiful subject of Teichmüller curves. Finally the authors want to thank the anonymous referees for a number of suggestions which helped improve the exposition of the paper.

During the preparation of this work the first author is partially supported by the NSF grant DMS-1101153 (transferred as DMS-1200329). The second author is partially supported by the ERC-StG 257137. 


\section{Background on moduli spaces}

\subsection{Strata of $\Omega \mathcal{M}_{g}$ and hyperelliptic loci}

Let $\Omega \mathcal{M}_{g}$ denote the vector bundle of holomorphic one-forms over the moduli space $\mathcal{M}_{g}$ of genus $g$ curves minus the zero section and let $\mathbb{P} \Omega \mathcal{M}_{g}$ denote the associated projective bundle. The spaces $\Omega \mathcal{M}_{g}$ and $\mathbb{P} \Omega \mathcal{M}_{g}$ are stratified according to the zeros of one-forms. For $m_{i} \geq 1$ and $\sum_{i=1}^{k} m_{i}=2 g-2$, let $\Omega \mathcal{M}_{g}\left(m_{1}, \ldots, m_{k}\right)$ denote the stratum parameterizing one-forms that have $k$ distinct zeros of order $m_{1}, \ldots, m_{k}$. Denote by $\overline{\mathcal{M}}_{g}$ the Deligne-Mumford compactification of $\mathcal{M}_{g}$. The boundary of $\overline{\mathcal{M}}_{g}$ parameterizes stable nodal curves, where the stability means the dualizing sheaf of the curve is ample, or equivalently, the normalization of any rational component needs to possess at least three special points coming from the inverse images of the nodes. The bundle of holomorphic one-forms extends over $\overline{\mathcal{M}}_{g}$, parameterizing stable oneforms or equivalently sections of the dualizing sheaf. We denote the total space of this extension by $\Omega \overline{\mathcal{M}}_{g}$.

Points in $\Omega \mathcal{M}_{g}$, called flat surfaces, are usually written as $(X, \omega)$ for a one-form $\omega$ on $X$. For a stable curve $X$, denote the dualizing sheaf by $\omega_{X}$. We will stick to the notation that points in $\Omega \overline{\mathcal{M}}_{g}$ are given by a pair $(X, \omega)$ with $\omega \in H^{0}\left(X, \omega_{X}\right)$.

For $d_{i} \geq-1$ and $\sum_{i=1}^{s} d_{i}=4 g-4$, let $\mathcal{Q}\left(d_{1}, \ldots, d_{s}\right)$ denote the moduli space of quadratic differentials that have $s$ distinct zeros or poles of order $d_{1}, \ldots, d_{s}$. The condition $d_{i} \geq-1$ ensures that the quadratic differentials in $\mathcal{Q}\left(d_{1}, \ldots, d_{S}\right)$ have at most simple poles. Namely, $\mathcal{Q}\left(d_{1}, \ldots, d_{s}\right)$ parameterizes pairs $(X, q)$ of a Riemann surface $X$ and a meromorphic section $q$ of $\omega_{X}^{\otimes 2}$ with the prescribed type of zeros and poles. Pairs $(X, q)$ are called half-translation surfaces. They appear occasionally to provide examples via the following construction.

If the quadratic differential is not a global square of a one-form, there is a natural double covering $\pi: Y \rightarrow X$ such that $\pi^{*} q=\omega^{2}$. This covering is ramified precisely at the zeros of odd order of $q$ and at its poles. It gives a map

$$
\phi: \mathcal{Q}\left(d_{1}, \ldots, d_{s}\right) \rightarrow \Omega \mathcal{M}_{g}\left(m_{1}, \ldots, m_{k}\right),
$$

where the signature $\left(m_{1}, \ldots, m_{k}\right)$ is determined by the ramification type. Indeed $\phi$ is an immersion (see Kontsevich and Zorich [33, Lemma 1]).

There are two cases where the domain and the range of the map $\phi$ have the same dimension:

$$
\begin{gathered}
\mathcal{Q}\left(-1^{2 g+1}, 2 g-3\right) \rightarrow \Omega \mathcal{M}_{g}(2 g-2), \\
\mathcal{Q}\left(-1^{2 g+2}, 2 g-2\right) \rightarrow \Omega \mathcal{M}_{g}(g-1, g-1) ;
\end{gathered}
$$


see [33, page 637]. In both cases we call the image a component of hyperelliptic flat surfaces of the corresponding stratum of Abelian differentials. Note that for both cases the domain of $\phi$ parameterizes genus zero curves. More generally, if the domain of $\phi$ parameterizes genus zero curves, we call the image a locus of hyperelliptic flat surfaces in the corresponding stratum. These loci are often called hyperelliptic loci, for example in $[33 ; 15]$. We prefer to reserve hyperelliptic locus for the subset of $\mathcal{M}_{g}$ (or its closure in $\overline{\mathcal{M}}_{g}$, see also Section 2.5) parameterizing hyperelliptic curves and thus specify with 'flat surfaces' if we speak of subsets of $\Omega \mathcal{M}_{g}$.

\subsection{Spin structures and connected components of strata}

A spin structure (or theta characteristic) on a smooth curve $X$ is a line bundle $\mathcal{L}$ whose square is the canonical bundle, ie $\mathcal{L}^{\otimes 2} \sim K_{X}$. The parity of a spin structure is given by $h^{0}(\mathcal{L}) \bmod 2$. This parity is well-known to be deformation invariant. There is a notion of spin structure on a stable curve, extending the smooth case (see Cornalba [10], also recalled by Farkas and Verra in [20, Section 1]). We only need the following consequence. The moduli space of spin curves $\overline{\mathcal{S}}_{g}$ parameterizes pairs $(X, \eta)$, where $\eta$ is a theta characteristic of $X$. It has two components $\overline{\mathcal{S}}_{g}^{-}$and $\overline{\mathcal{S}}_{g}^{+}$distinguished by the parity of the spin structure. The spin structures on stable curves are defined such that the morphisms $\pi: \overline{\mathcal{S}}_{g}^{-} \rightarrow \overline{\mathcal{M}}_{g}$ and $\pi: \overline{\mathcal{S}}_{g}^{+} \rightarrow \overline{\mathcal{M}}_{g}$ are finite of degree $2^{g-1}\left(2^{g}-1\right)$ and $2^{g-1}\left(2^{g}+1\right)$, respectively, cf loc cit.

Recall the classification of connected components of strata in $\Omega \mathcal{M}_{g}$ by Kontsevich and Zorich [33, Theorem 1 on page 639].

Theorem 2.1 [33] The strata of $\Omega \mathcal{M}_{g}$ have up to three connected components, distinguished by the parity of the spin structure and by being hyperelliptic or not. For $g \geq 4$, the strata $\Omega \mathcal{M}_{g}(2 g-2)$ and $\Omega \mathcal{M}_{g}(2 k, 2 k)$ with an integer $k=(g-1) / 2$ have three components, the component of hyperelliptic flat surfaces and two components with odd or even parity of the spin structure but not consisting exclusively of hyperelliptic curves.

The stratum $\Omega \mathcal{M}_{3}(4)$ has two components, $\Omega \mathcal{M}_{3}(4)^{\text {hyp }}$ and $\Omega \mathcal{M}_{3}(4)^{\text {odd }}$. The stratum $\Omega \mathcal{M}_{3}(2,2)$ also has two components, $\Omega \mathcal{M}_{3}(2,2)^{\text {hyp }}$ and $\Omega \mathcal{M}_{3}(2,2)^{\text {odd }}$.

Each stratum $\Omega \mathcal{M}_{g}\left(2 k_{1}, \ldots, 2 k_{r}\right)$ for $r \geq 3$ or $r=2$ and $k_{1} \neq(g-1) / 2$ has two components determined by even and odd spin structures.

Each stratum $\Omega \mathcal{M}_{g}(2 k-1,2 k-1)$ for $k \geq 2$ has two components, the component of hyperelliptic flat surfaces $\Omega \mathcal{M}_{g}(2 k-1,2 k-1)^{\text {hyp }}$ and the other component $\Omega \mathcal{M}_{g}(2 k-1,2 k-1)^{\text {non-hyp }}$. In all the other cases, the stratum is connected. 
Consider the partition $(2, \ldots, 2)$. For $(X, \omega) \in \Omega \mathcal{M}_{g}(2, \ldots, 2)^{\text {odd }}$ with $\operatorname{div}(\omega)=$ $2 \sum_{i=1}^{g-1} p_{i}$, the line bundle $\eta=\mathcal{O}_{X}\left(\sum_{i=1}^{g-1} p_{i}\right)$ is an odd theta characteristic. Therefore, we have a natural morphism

$$
f: \Omega \mathcal{M}_{g}(2, \ldots, 2)^{\text {odd }} \rightarrow \overline{\mathcal{S}}_{g}^{-} .
$$

Note that $f$ contracts the locus where $h^{0}(\eta)>1$. Similarly one can define such a morphism for even spin structures.

\subsection{Picard groups of moduli spaces}

Let $\mathcal{M}_{g, n}$ be the moduli space (treated as a stack instead of the course moduli scheme) of genus $g$ curves with $n$ ordered marked points and let $\mathcal{M}_{g,[n]}$ be the moduli space of genus $g$ curves with $n$ unordered marked points. We write Pic $(\cdot)$ for the rational Picard group $\operatorname{Pic}_{\text {fun }}(\cdot)_{\mathbb{Q}}$ of a moduli stack (see eg [27, Section 3.D] for more details).

We fix some standard notation for elements in the Picard group. Let $\lambda$ denote the first Chern class of the Hodge bundle. Let $\delta_{i}, i=1, \ldots,\lfloor g / 2\rfloor$ be the boundary divisor of $\overline{\mathcal{M}}_{g}$ whose generic element is a smooth curve of genus $i$ joined at a node to a smooth curve of genus $g-i$. The generic element of the boundary divisor $\delta_{0}$ is an irreducible nodal curve of geometric genus $g-1$. In the literature sometimes $\delta_{0}$ is denoted by $\delta_{\text {irr }}$. We write $\delta$ for the total boundary class.

For moduli spaces with marked points we denote by $\omega_{\text {rel }}$ the relative dualizing sheaf of $\overline{\mathcal{M}}_{g, 1} \rightarrow \overline{\mathcal{M}}_{g}$ and $\omega_{i \text {,rel }}$ its pullback to $\overline{\mathcal{M}}_{g, n}$ via the map forgetting all but the $i$-th marked point. For a set $S \subset\{1, \ldots, n\}$ we let $\delta_{i ; S}$ denote the boundary divisor whose generic element is a smooth curve of genus $i$ joined at a node to a smooth curve of genus $g-i$ and the sections in $S$ lying on the first component.

Theorem 2.2 The rational Picard group of $\overline{\mathcal{M}}_{g}$ is generated by $\lambda$ and the boundary classes $\delta_{i}, i=0, \ldots,\lfloor g / 2\rfloor$.

More generally, the rational Picard group of $\overline{\mathcal{M}}_{g, n}$ is generated by $\lambda, \omega_{i, \text { rel }}, i=1, \ldots, n$, by $\delta_{0}$ and by $\delta_{i ; S}, i=0, \ldots,\lfloor g / 2\rfloor$, where $|S|>1$ if $i=0$ and $1 \in S$ if $i=g / 2$.

The above theorem essentially follows from Harer's result $H^{0}\left(\overline{\mathcal{M}}_{g}, \mathbb{Q}\right) \cong \mathbb{Q}[\lambda][24]$. The reader may also refer to Mumford [39] for a comparison between the rational Picard group of the coarse moduli scheme and of the moduli stack, as well as Arbarello and Cornalba [1] for the Picard group with integral coefficients.

Alternatively, define $\psi_{i} \in \operatorname{Pic}\left(\overline{\mathcal{M}}_{g, n}\right)$ to be the class with value $-\pi_{*}\left(\sigma_{i}^{2}\right)$ on any family of stable genus $g$ curves $\pi: \mathcal{X} \rightarrow C$ with section $\sigma_{i}$ corresponding to the $i$-th marked 
point. By induction on $n$, we have the relation

$$
\omega_{i, \text { rel }}=\psi_{i}-\sum_{i \in S} \delta_{0 ; S}
$$

see eg [1, page 161] and Logan [34, page 108]. Consequently, a generating set of $\operatorname{Pic}\left(\overline{\mathcal{M}}_{g, n}\right)$ can also be formed by the $\psi_{i}, \lambda$ and boundary classes.

For a divisor class $D=a \lambda-\sum_{i=0}^{\lfloor g / 2\rfloor} b_{i} \delta_{i}$ in $\operatorname{Pic}\left(\overline{\mathcal{M}}_{g}\right)$, define its slope to be

$$
s(D)=\frac{a}{b_{0}} \text {. }
$$

For our purpose the higher boundary divisors need not to be considered, as Teichmüller curves generated by Abelian differentials do not intersect $\delta_{i}$ for $i>0$ (see Corollary 3.2).

\subsection{Linear series on curves}

Many divisors on moduli spaces of curves are related to the geometry of linear series. Here we review some basic properties of linear series on curves (see Arbarello, Cornalba, Griffiths and Harris [2] for a comprehensive introduction).

Let $X$ be a genus $g$ curve and $\mathcal{L}$ a line bundle of degree $d$ on $X$. Denote by $|\mathcal{L}|$ the linear system parameterizing sections of $\mathcal{L}$ mod scalars, ie

$$
|\mathcal{L}|=\left\{\operatorname{div}(s) \mid s \in H^{0}(\mathcal{L})\right\} .
$$

If $h^{0}(\mathcal{L})=n$, then $|\mathcal{L}| \cong \mathbb{P}^{n-1}$. For a (projective) $r$-dimensional linear subspace $V$ of $|\mathcal{L}|$, call $(\mathcal{L}, V)$ a linear series $g_{d}^{r}$. If $\mathcal{L} \sim \mathcal{O}_{X}(D)$ for a divisor $D$ on $X$, we also denote by $\left|\mathcal{O}_{X}(D)\right|$ or simply by $|D|$ the linear system.

If all divisors parameterized in a linear series contain a common point $p$, then $p$ is called a base point. Otherwise, this linear series is called base-point-free. A base-pointfree $g_{d}^{r}$ induces a morphism $X \rightarrow \mathbb{P}^{r}$. The divisors in this $g_{d}^{r}$ correspond to (the pullback of) hyperplane sections of the image curve. For instance, a hyperelliptic curve admits a $g_{2}^{1}$, ie a double cover of $\mathbb{P}^{1}$. The following fact will be used frequently when we prove the disjointness of Teichmüller curves with a geometrically defined divisor.

Proposition 2.3 A point $p$ is not a base point of a linear system $|\mathcal{L}|$ if and only if $h^{0}(\mathcal{L})-1=h^{0}(\mathcal{L}(-p))$, where $\mathcal{L}(-p)=\mathcal{L} \otimes \mathcal{O}_{X}(-p)$.

Proof By the exact sequence

$$
0 \rightarrow \mathcal{L}(-p) \rightarrow \mathcal{L} \rightarrow \mathcal{O}_{p} \rightarrow 0,
$$


we know $h^{0}(\mathcal{L}(-p))$ is either equal to $h^{0}(\mathcal{L})$ or $h^{0}(\mathcal{L})-1$. The former happens if and only if every section of $|\mathcal{L}|$ vanishes at $p$, in other words, if and only if $p$ is a base point of $|\mathcal{L}|$.

The canonical linear system is a $g_{2 g-2}^{g-1}$, which induces an embedding to $\mathbb{P}^{g-1}$ for a nonhyperelliptic curve. The image of this embedding is called a canonical curve. Let $D$ be an effective divisor of degree $d$ on $X$. Denote by $\overline{\phi_{K}(D)}$ the linear subspace in $\mathbb{P}^{g-1}$ spanned by the images of points in $D$ under the canonical map $\phi_{K}$. The following geometric version of the Riemann-Roch theorem is useful for the study of canonical curves (see [2, page 12] for more details).

Theorem 2.4 (Geometric Riemann-Roch) In the above setting, we have

$$
\operatorname{dim}|D|=d-1-\operatorname{dim} \overline{\phi_{K}(D)} .
$$

We will focus on the geometry of canonical curves of low genus. Curves of genus 2 are always hyperelliptic. For nonhyperelliptic curves of genus 3, their canonical images correspond to plane quartics.

For $g=4$, a nonhyperelliptic canonical curve $X$ in $\mathbb{P}^{3}$ is a complete intersection cut out by a quadric and a cubic. Any divisor $D=p+q+r$ in a $g_{3}^{1}$ of $X$ spans a line in $\mathbb{P}^{3}$, by Geometric Riemann-Roch. This line intersects $X$ at $p, q, r$, hence it is contained in the quadric by Bézout. If the quadric is smooth, it is isomorphic to $\mathbb{P}^{1} \times \mathbb{P}^{1}$. It has two families of lines, called two rulings. Any line in a ruling intersects $X$ at three points (with multiplicity), hence $X$ has two different linear systems $g_{3}^{1}$ corresponding to the two rulings. If the quadric is singular, then it is a quadric cone with a unique ruling, hence $X$ has a unique $g_{3}^{1}$.

For $g=5$, a general canonical curve is cut out by three quadric hypersurfaces in $\mathbb{P}^{4}$ and it does not have any $g_{3}^{1}$. On the other hand, a genus 5 curve with a $g_{3}^{1}$, ie a trigonal curve, has canonical image contained in a cubic scroll surface, which is a ruled surface of degree 3 in $\mathbb{P}^{4}$. By Geometric Riemann-Roch, divisors in the $g_{3}^{1}$ span rulings that sweep out the surface (see eg Reid [40, Section 2.10]).

Recall that on a nodal curve $X$, Serre duality and Riemann-Roch hold with the dualizing sheaf $\omega_{X}$ in place of the canonical bundle (see eg [27, Section 3.A] for more details). We also need the following generalized Clifford's theorem for DeligneMumford stable curves (see eg [2, page 107] for the case of smooth curves and Caporaso [6, Theorems 3.3, 4.11] for the remaining cases). Following [6, Section 2.1] let $\delta_{Z}=Z \cdot Z^{c}$ be the number of intersection points of $Z$ with its complement and 
$w_{Z}=2 g(Z)-2+\delta_{Z}$. A divisor $D$ of degree $d$ on a stable curve $X$ is balanced, if for every irreducible component $Z \subset X$ we have

$$
d \frac{w_{Z}}{2 g-2}-\frac{\delta_{Z}}{2} \leq \operatorname{deg}\left(\left.D\right|_{Z}\right) \leq d \frac{w_{Z}}{2 g-2}-\frac{\delta_{Z}}{2} .
$$

Theorem 2.5 (Clifford's theorem) Let $X$ be a stable curve and $D$ an effective divisor on $X$ with $\operatorname{deg}(D) \leq 2 g-1$. Then we have

$$
h^{0}\left(\mathcal{O}_{X}(D)\right)-1 \leq \operatorname{deg}(D) / 2
$$

if one of the following conditions holds: (i) $X$ is smooth; (ii) $X$ has at most two components and $D$ is balanced; (iii) $X$ does not have separating nodes, $\operatorname{deg}(D) \leq 4$ and $D$ is balanced.

Finally we need to consider the canonical system of a stable curve associated to its dualizing sheaf. This will help us discuss the boundary of Teichmüller curves. Recall the dual graph of a nodal curve whose vertices correspond to its irreducible components and edges correspond to intersections of these components. A graph is called $n$-connected if one has to remove at least $n$ edges to disconnect the graph. The following fact characterizes canonical maps of stable curves based on the type of their dual graphs (see Hassett [29, Proposition 2.3]).

Proposition 2.6 Let $X$ be a stable curve of genus greater than or equal to 2. Then the canonical linear system $\left|\omega_{X}\right|$ is base point free (resp. very ample) if and only if the dual graph of $X$ is two-connected (resp. three-connected and $X$ is not in the closure of the locus of hyperelliptic curves).

\subsection{Special divisors on moduli spaces}

In the application for Teichmüller curves generated by flat surfaces we do not care about the coefficients of $\delta_{i}$ for $i \geq 1$ in the divisor classes in $\operatorname{Pic}\left(\overline{\mathcal{M}}_{g}\right)$, since Teichmüller curves do not intersect those components (see Corollary 3.2). As shorthand, we use $\delta_{\text {other }}$ to denote some linear combination of $\delta_{i}$ for $i \geq 1$. Similarly, in $\overline{\mathcal{M}}_{g, n}$ we use $\delta_{\text {other }}$ to denote some linear combination of all boundary divisors but $\delta_{0}$. By the same reason we do not distinguish between $\omega_{i \text {,rel }}$ and $\psi_{i}$ for a divisor class, since they only differ by boundary classes in $\delta_{\text {other }}$.

The hyperelliptic locus in $\overline{\mathcal{M}}_{3}$ Denote by $H \subset \overline{\mathcal{M}}_{g}$ the closure of locus of genus $g$ hyperelliptic curves. We call $H$ the hyperelliptic locus in $\overline{\mathcal{M}}_{g}$. Note that $H$ is a divisor if and only if $g=3$. A stable curve $X$ lies in the boundary of $H$ if there is an 
admissible cover of degree two $\tilde{X} \rightarrow \mathbb{P}^{1}$, for some nodal curve $\tilde{X}$ whose stabilization is $X$. We refer to [27, Section 3.G] for an excellent introduction to admissible covers. The class of the hyperelliptic locus $H \subset \overline{\mathcal{M}}_{3}$ calculated eg in [27, page 188] is given as follows:

$$
H=9 \lambda-\delta_{0}-3 \delta_{1}
$$

hence it has slope $s(H)=9$.

Divisors of Weierstrass points Let $W \subset \overline{\mathcal{M}}_{g, 1}$ be the divisor parameterizing a curve with a Weierstrass point. In [12, (2.0.12) on page 328], Cukierman calculated the class of $W$ for all $g$, which specializes as follows:

$$
W=6 \omega_{\text {rel }}-\lambda-\delta_{\text {other }}, \quad \text { for } g=3 .
$$

The theta-null divisor Consider the divisor $\Theta \subset \overline{\mathcal{M}}_{g, 1}$ parameterizing $(X, p)$ such that $X$ admits an odd theta characteristic whose support contains $p$. The class of $\Theta$ was calculated by Farkas in [19, Theorem 0.2], which specializes as follows:

$$
\Theta=30 \lambda+60 \omega_{\text {rel }}-4 \delta_{0}-\delta_{\text {other }}, \quad \text { for } g=4 \text {. }
$$

The Brill-Noether divisors The Brill-Noether locus $B N_{d}^{r}$ in $\overline{\mathcal{M}}_{g}$ parameterizes curves $X$ that possesses a $g_{d}^{r}$. If the Brill-Noether number is -1 , ie

$$
\rho(g, r, d)=g-(r+1)(g-d+r)=-1,
$$

then $B N_{d}^{r}$ is indeed a divisor. We remark that nowadays $\mathcal{M}_{g, d}^{r}$ is more commonly used to denote the Brill-Noether divisors, but we decide to reserve $\mathcal{M}$ for the moduli space only.

There are pointed versions of this divisor. Let $\underline{w}=\left(w_{1}, \ldots, w_{n}\right)$ be a tuple of integers. Let $B N_{d, \underline{w}}^{r}$ be the locus in $\overline{\mathcal{M}}_{g, n}$ of pointed curves $\left(X, p_{1}, \ldots, p_{n}\right)$ with a line bundle $\mathcal{L}$ of degree $d$ such that $\mathcal{L}$ admits a $g_{d}^{r}$ and $h^{0}\left(\mathcal{L}\left(-\sum w_{i} p_{i}\right)\right) \geq r$. This Brill-Noether locus is a divisor, if the generalized Brill-Noether number is -1 , ie

$$
\rho(g, r, d, \underline{w})=g-(r+1)(g-d+r)-r(|\underline{w}|-1)=-1 .
$$

The hyperelliptic divisor and the Weierstrass divisor could also be interpreted as BrillNoether divisors, but we stick to the traditional notation for them.

The class of these pointed divisors has been calculated in many special cases, in particular in [34] and later by Farkas in [18]. We collect the results that are needed here. 
The class of the classical Brill-Noether divisor for $r=1$ was calculated by Harris and Mumford in [28, page 24], and in particular

$$
B N_{3}^{1}=8 \lambda-\delta_{0}-\delta_{\text {other }}, \quad \text { for } g=5 \text {. }
$$

If $|\underline{w}|=g=d$ and $r=1$ the class of the Brill-Noether divisor was calculated in [34, Theorem 5.4]. It has class

$$
B N_{g, \underline{w}}^{1}=-\lambda+\sum_{i=1}^{k} \frac{w_{i}\left(w_{i}+1\right)}{2} \omega_{i, \text { rel }}-\delta_{\text {other }}
$$

In particular for $\underline{w}=(1,2)$, it specializes as follows:

$$
B N_{3,(1,2)}^{1}=-\lambda+\omega_{1, \text { rel }}+3 \omega_{2, \text { rel }}-\delta_{\text {other }}, \quad \text { for } g=3 \text {, }
$$

For $\underline{w}=(1,1,2)$, it specializes as follows:

$$
B N_{4,(1,1,2)}^{1}=-\lambda+\omega_{1, \mathrm{rel}}+\omega_{2, \mathrm{rel}}+3 \omega_{3, \mathrm{rel}}-\delta_{\mathrm{other}}, \quad \text { for } g=4
$$

If $r=1$ and $\underline{w}=(2)$, the class of the divisor was also calculated in [34]. It specializes to

$$
B N_{3,(2)}^{1}=4 \omega_{\text {rel }}+8 \lambda-\delta_{0}-\delta_{\text {other }}, \quad \text { for } g=4 \text {. }
$$

If all $w_{i}=1$ and $n=r+1$ the Brill-Noether divisor specializes to the divisor Lin calculated in [18, Section 4.2]. In particular [18, Theorem 4.6] gives

$$
\operatorname{Lin}_{3}^{1}=B N_{3,(1,1)}^{1}=-\omega_{1, \mathrm{rel}}-\omega_{2, \mathrm{rel}}+8 \lambda-\delta_{0}-\delta_{\text {other }}, \quad \text { for } g=4 .
$$

Generalizing the calculation of Logan for $r=1$ and $n=1$ to arbitrary weight $w_{1}$, one obtains the divisor called Nfold $_{d}^{1}(1)$ in the proof of [18, Theorem 4.9]. From the proof one deduces

$$
\operatorname{Nfold}_{4}^{1}(1)=B N_{4,(3)}^{1}=7 \lambda+15 \omega_{\text {rel }}-\delta_{0}-\delta_{\text {other }}, \quad \text { for } g=5 .
$$

Nfold(1) is a degeneration of the divisor Nfold in [18]. A partial degeneration is $\operatorname{Nfold}(2)=B N_{4,(1,2)}^{1}$ in $\overline{\mathcal{M}}_{5,2}$. It has class

$$
\operatorname{Nfold}_{4}^{1}(2)=B N_{4,(1,2)}^{1}=7 \lambda+7 \omega_{1, \mathrm{rel}}+2 \omega_{2, \mathrm{rel}}-\delta_{0}-\delta_{\mathrm{other}}, \quad \text { for } g=5
$$

Since this divisor class was not explicitly written out in [18], below we give a proof.

Proof of (10) Using the same logic in the proof of [18, Theorems 4.6,4.9], $\lambda, \delta_{0}, \psi_{1}$ have nonvarying coefficients in $\mathrm{Nfold}_{4}^{1}$, which is $B N_{4,(1,1,1)}^{1}$ in our notation, and in $\mathrm{Nfold}_{4}^{1}(2)$. Hence we have

$$
\operatorname{Nfold}_{4}^{1}(2)=7 \lambda-\delta_{0}+2 \psi_{1}+c \psi_{2}-e \delta_{0 ;\{1,2\}}-\delta_{\text {other }}
$$


We have to take $\delta_{0 ;\{1,2\}}$ into account, because the test curves used below intersect $\delta_{0 ;\{1,2\}}$. Let $X$ be a general curve of genus five. Take a fixed general point $x_{2}$ on $X$ and move another point $x_{1}$ along $C$. Call this family $B_{1}$. We have

$$
\begin{gathered}
B_{1} \cdot \lambda=0, \quad B_{1} \cdot \delta_{0}=0, \quad B_{1} \cdot \delta_{0 ;\{1,2\}}=1, \\
B_{1} \cdot \psi_{1}=9, \quad B_{1} \cdot \psi_{2}=1 .
\end{gathered}
$$

The intersection number $B_{1} \cdot \mathrm{Nfold}_{4}^{1}(2)$ can be calculated using [34, Proposition 3.4] by setting $a_{1}=2, a_{2}=1, g=5, h=1$, and it equals 10 . Note that Logan counts the number of pairs $\left(p_{2}, q_{1}\right)$, which equals 5 , but for our purpose $x_{1}$ can be either $p_{2}$ or $q_{1}$, so we double the counting. We thus obtain a relation

$$
c-e+8=0 .
$$

Now fix a general point $x_{1}$ and move another point $x_{2}$ along $X$. Call this family $B_{2}$. We have

$$
\begin{gathered}
B_{2} \cdot \lambda=0, \quad B_{2} \cdot \delta_{0 ;\{1,2\}}=1, \\
B_{2} \cdot \psi_{1}=1, \quad B_{2} \cdot \psi_{2}=9 .
\end{gathered}
$$

The intersection number $B_{2} \cdot \mathrm{Nfold}(2)$ can also be calculated using [34, Proposition 3.4] by setting $a_{1}=1, a_{2}=2, g=5, h=1$, and it equals 50 . This equals Logan's counting, since in the pair $\left(p_{2}, q_{1}\right)$ now $p_{2}$ has weight 2 , which distinguishes it from $q_{1}$. We then obtain another relation

$$
9 c-e-48=0 .
$$

Combining the two relations we conclude that $c=7, e=15$, which completes the proof.

Gieseker-Petri divisors Consider a linear series $(\mathcal{L}, V) \in G_{d}^{r}(X)$ for a linear subspace $V \subset H^{0}(\mathcal{L})$ of dimension $r+1, \operatorname{deg}(\mathcal{L})=d$ and the multiplication map

$$
\mu: V \otimes H^{0}\left(\omega_{X} \otimes \mathcal{L}^{-1}\right) \rightarrow H^{0}\left(\omega_{X}\right) .
$$

Define the Gieseker-Petri locus

$G P_{g, d}^{r}=\left\{[X] \in \overline{\mathcal{M}}_{g}, \exists\right.$ base-point-free $(\mathcal{L}, V) \in G_{d}^{r}(X)$ such that $\mu$ is not injective $\}$.

The divisor class of the Gieseker-Petri locus in the case $r=1$ was calculated by Eisenbud and Harris in [14, Theorem 2]. It specializes to

$$
G P=17 \lambda-2 \delta_{0}+\delta_{\text {other }}, \quad \text { for } g=4 .
$$


Alternatively, one can describe $G P$ in $\mathcal{M}_{4}$ as follows. The canonical image of a genus 4 nonhyperelliptic curve is contained in a quadric surface in $\mathbb{P}^{3}$. Then $G P$ is the closure of the locus where this quadric is singular (see eg [2, page 196]).

\section{Teichmüller curves and their boundary points}

We quickly recall the definition of Teichmüller curves and of square-tiled surfaces which serve as main examples. New results on the boundary behavior of Teichmüller curves needed later are collected in Section 3.3.

\subsection{Teichmüller curves as fibered surfaces}

A Teichmüller curve $C \rightarrow \mathcal{M}_{g}$ is an algebraic curve in the moduli space of curves that is totally geodesic with respect to the Teichmüller metric. There exists a finite unramified cover $B \rightarrow C$ such that the monodromies around the 'punctures' $\bar{B} \backslash B$ are unipotent and such that the universal family over some level covering of $\mathcal{M}_{g}$ pulls back to a family of curves $f: \mathcal{X} \rightarrow B$. We denote by $f: \overline{\mathcal{X}} \rightarrow \bar{B}$ a relatively minimal semistable model of a fibered surface of fiber genus $g$ with smooth total space. Let $\Delta \subset \bar{B}$ be the set of points with singular fibers, hence $B=\bar{B} \backslash \Delta$. See eg the second author [36] for more on this setup. By a further finite unramified covering (outside $\Delta$ ) we may suppose that the zeros of $\omega$ on $X$ extend to sections $\sigma_{i}$ of $f$. We denote by $S_{i} \subset \overline{\mathcal{X}}$ the images of these sections.

Teichmüller curves arise as the $\mathrm{SL}_{2}(\mathbb{R})$-orbit of special flat surfaces or half-translation surfaces, called Veech surfaces. We deal here with the first case only and denote by $(X, \omega)$ a generating flat surface, if its $\mathrm{SL}_{2}(\mathbb{R})$ orbit gives rise to a Teichmüller curve. Teichmüller curves come with a uniformization $C=\mathbb{H} / \operatorname{SL}(X, \omega)$, where $\operatorname{SL}(X, \omega)$ is the affine group (or Veech group) of the flat surface $(X, \omega)$. Let $K=\mathbb{Q}(\operatorname{tr}(\gamma), \gamma \in$ $\operatorname{SL}(X, \omega))$ denote the trace field of the affine group and let $L / \mathbb{Q}$ denote the Galois closure of $K / \mathbb{Q}$.

The variation of Hodge structure (VHS) over a Teichmüller curve decomposes into sub-VHS

$$
R^{1} f_{*} \mathbb{C}=\left(\oplus_{\sigma \in \operatorname{Gal}(L / \mathbb{Q}) / \operatorname{Gal}(K / \mathbb{Q})} \mathbb{L}^{\sigma}\right) \oplus \mathbb{M},
$$

where $\mathbb{L}$ is the VHS with the standard 'affine group' representation, $\mathbb{L}^{\sigma}$ are the Galois conjugates and $\mathbb{M}$ is just some representation ([36, Proposition 2.4]). One of the purposes of our work is to shed some light on what possibilities for the numerical data of $\mathbb{M}$ can occur. 


\subsection{Square-tiled surfaces}

A square-tiled surface is a flat surface $(X, \omega)$, where $X$ is obtained as a covering of a torus ramified over one point only and $\omega$ is the pullback of a holomorphic one-form on the torus. It is well-known that in this case $\operatorname{SL}(X, \omega)$ is commensurable to $\operatorname{SL}_{2}(\mathbb{Z})$, hence $\mathbb{L}$ has no Galois conjugates or equivalently, the rank of $\mathbb{M}$ is $2 g-2$.

In order to specify a square-tiled surface covered by $d$ squares, it suffices to specify the monodromy of the covering. Take a standard torus $E$ by identifying via affine translation the two pairs of parallel edges of the unit square $[0,1] \times[0, i]$. Consider the closed, oriented paths $u=[0,1]$ on the horizontal axis and $r=[0, i]$ on the vertical axis. The indices $u$ and $r$ correspond to 'up' and 'right', respectively. Note that $u$ and $r$ form a basis of $\pi_{1}(E, b)$, where $b$ is a base point in $E$. Going along $u$ and $r$ induces two permutations $\left(\pi_{u}, \pi_{r}\right)$ on the $d$ sheets of a degree $d$ cover of $E$. Hence $\pi_{u}, \pi_{r}$ can be regarded as elements in the symmetric group $S_{d}$. Conversely, given such a pair $\left(\pi_{u}, \pi_{r}\right)$, one can construct a degree $d$ cover of $E$ (possibly disconnected) ramified over one point only. The domain of the covering is connected if and only if the subgroup in $S_{d}$ generated by $\pi_{u}, \pi_{r}$ acts transitively on the $d$ letters. Moreover, the ramification profile over $b$ is determined by the commutator $\pi_{u}^{-1} \pi_{r}^{-1} \pi_{u} \pi_{r}$.

The surface in Figure 1 corresponds to a degree 5, genus 2, connected cover of the standard torus: It is easy to see that the monodromy permutations for this square-tiled

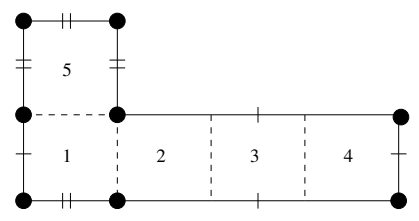

Figure 1: A square-tiled surface of degree 5 and genus 2

surface are given by $\left(\pi_{u}=(15)(2)(3)(4), \pi_{r}=(1234)(5)\right)$. Here a cycle $\left(a_{1} \ldots a_{k}\right)$ means the permutation sends $a_{i}$ to $a_{i+1}$ for $1 \leq i \leq k-1$ and sends $a_{k}$ back to $a_{1}$. One can check that $\pi_{u}^{-1} \pi_{r}^{-1} \pi_{u} \pi_{r}=(154)(2)(3)$. Therefore, the corresponding covering has a unique ramification point marked by $\bullet$ with ramification order $2=3-1$ arising from the length 3 cycle, since locally the three sheets labeled by 1, 5, 4 get permuted at that point. By Riemann-Hurwitz, the domain of the covering has genus equal to 2 . The pullback of $d z$ from $E$ is a one-form in the stratum $\Omega \mathcal{M}_{2}(2)$.

Based on the monodromy data, one can directly calculate the Siegel-Veech constant as well as the sum of Lyapunov exponents (introduced in the next section) for a Teichmüller curve generated by a square-tiled surface (see [15]). Later on we will 
use square-tiled surfaces to produce examples of Teichmüller curves that have varying sums of Lyapunov exponents.

\subsection{Properties of Teichmüller curves}

Here we collect the properties of the boundary points of Teichmüller curves that are needed in the proofs in the subsequent sections. We will use $\bar{C}$ to denote the closure of a Teichmüller curve $C$ in the compactified moduli space.

Let $\mu$ be a partition of $2 g-2$. If $\mu^{\prime}$ is another partition and if it can be obtained from $\mu$ by successively combining two entries into one, we say that $\mu^{\prime}$ is a degeneration of $\mu$. For instance, $(2,6)$ is a degeneration of $(1,1,3,3)$. Geometrically speaking, combining two entries $i, j$ corresponds to merging two zeros of order $i, j$ into a single zero of order $i+j$.

Proposition 3.1 Suppose $C$ is a Teichmüller curve generated by a flat surface in $\Omega \mathcal{M}_{g}(\mu)$ and let $\mu^{\prime}$ be a degeneration of $\mu$. Then $\bar{C}$ in $\mathbb{P} \Omega \overline{\mathcal{M}}_{g}(\mu)$ is disjoint from $\mathbb{P} \Omega \overline{\mathcal{M}}_{g}\left(\mu^{\prime}\right)$.

Proof The claim is obvious over the interior of the moduli space. We only need to check the disjointness over the boundary. The cusps of Teichmüller curves are obtained by applying the Teichmüller geodesic flow $\operatorname{diag}\left(e^{t}, e^{-t}\right)$ to the direction of the flat surface $(X, \omega)$ in which $(X, \omega)$ decomposes completely into cylinders. The stable surface at the cusp is obtained by 'squeezing' the core curves of these cylinders. This follows from the explicit description by Masur in [35]. Since the zeros of $\omega$ are located away from the core curves of the cylinders, the claim follows.

For a nodal curve, a node is called separating if removing it disconnects the curve.

Corollary 3.2 The section $\omega$ of the canonical bundle of each smooth fiber over a Teichmüller curve $C$ extends to a section $\omega_{\infty}$ of the dualizing sheaf for each singular fiber $X_{\infty}$ over the closure of a Teichmüller curve. The signature of zeros of $\omega_{\infty}$ is the same as that of $\omega$. Moreover, $X_{\infty}$ does not have separating nodes. In particular, $\bar{C}$ does not intersect $\delta_{i}$ for $i>0$.

Proof The first statement follows from the description in the preceding proof. The fact that $X_{\infty}$ does not have separating nodes is a consequence of the topological fact that a core curve of a cylinder can never disconnect a flat surface. It implies that $\bar{C}$ does not intersect the boundary divisors $\delta_{i}$ for $i>0$ on $\overline{\mathcal{M}}_{g}$, because by definition a curve parameterized in $\delta_{i}$ for $i>0$ possesses at least one separating node. 
Corollary 3.3 For Teichmüller curves generated by a flat surface in $\Omega \mathcal{M}_{g}(2 g-2)$ the degenerate fibers are irreducible.

For Teichmüller curves generated by a flat surface in $\Omega \mathcal{M}_{g}\left(k_{1}, k_{2}\right)$, with $k_{1} \geq k_{2}$ both odd, the degenerate fibers are irreducible or consist of two components of genus $g_{i}$ for $i=1,2$ joined at $n$ nodes for an odd number $n$ such that $2 g_{i}-2+n=k_{i}$.

Proof Let $X$ be a degenerate fiber and $Z$ a component of $X$. The dualizing sheaf of $X$ restricted to $Z$ has positive degree equal to $2 g_{Z}-2+\delta_{Z}$, where $\delta_{Z}$ is the intersection number of $Z$ with its complement in $X$. For the case $\Omega \mathcal{M}_{g}(2 g-2)$, by Corollary 3.2 it implies that $X$ only has one component, hence it is irreducible. For the case $\Omega \mathcal{M}_{g}\left(k_{1}, k_{2}\right)$, it implies that $X$ is either irreducible or has two components $Z_{1}, Z_{2}$. For the latter suppose $Z_{i}$ contains the $k_{i}$-fold zero. By assumption $2 g_{i}-2+\delta_{Z_{i}}=k_{i}$ is odd, hence $\delta_{Z_{1}}=\delta_{Z_{2}}=n$ is also odd.

Proposition 3.4 Let $C$ be a Teichmüller curve generated by a flat surface $(X, \omega)$ in $\Omega \mathcal{M}_{g}(\mu)$. Suppose an irreducible degenerate fiber $X_{\infty}$ over a cusp of $C$ is hyperelliptic. Then $X$ is hyperelliptic, hence the whole Teichmüller curve lies in the locus of hyperelliptic flat surfaces.

Moreover, if $\mu \in\{(4),(3,1),(6),(5,1),(3,3),(3,2,1),(8),(5,3)\}$ and $(X, \omega)$ is not hyperelliptic, then no degenerate fiber of the Teichmüller curve is hyperelliptic.

The last conclusion does not hold for all strata. For instance, Teichmüller curves generated by a nonhyperelliptic flat surface in the stratum $\Omega \mathcal{M}_{3}(2,1,1)$ always intersect the hyperelliptic locus at the boundary, as we will see later in the discussion for that stratum.

As motivation for the proof, recall why a Teichmüller curve generated by $(X, \omega)$ with $X$ hyperelliptic stays within the corresponding locus of hyperelliptic flat surfaces. The hyperelliptic involution acts as $(-1)$ on all one-forms, hence on $\omega$. In the flat coordinates of $X$ given by $\operatorname{Re}(\omega)$ and $\operatorname{Im}(\omega)$, the hyperelliptic involution acts by the matrix -Id. The Teichmüller curve is the $\mathrm{SL}_{2}(\mathbb{R})$-orbit of $(X, \omega)$ and $-\mathrm{Id}$ is in the center of $\mathrm{SL}_{2}(\mathbb{R})$. So if $(X, \omega)$ admits a hyperelliptic involution, so does $A \cdot(X, \omega)$ for any $A \in \mathrm{SL}_{2}(\mathbb{R})$.

Proof Suppose the stable model $X_{\infty}$ of the degenerate fiber is irreducible of geometric genus $h$ with $(g-h)$ pairs of points $\left(p_{i}, q_{i}\right)$ identified. This stable curve $X_{\infty}$ being hyperelliptic means that there exists a semistable curve birational to $X_{\infty}$ that admits a degree two admissible cover of the projective line. In terms of admissible covers, this is yet equivalent to require that the normalization $X_{n}$ of $X_{\infty}$ is branched at $2 h+2$ 
branch points over a main component (ie the image of the unique component not contracted under that passage to the stable model) with covering group generated by an involution $\phi$ and, moreover, for each of the $2(g-h)$ nodes there is a projective line intersecting $X_{n}$ in $p_{i}$ and $q_{i}=\phi\left(p_{i}\right)$ with two branch points.

In the flat coordinates of $X_{n}$ given by $\omega$, the surface consists of a compact surface $X_{0}$ with boundary of genus $h$ and $2(g-h)$ half-infinite cylinders (corresponding to the nodes) attached to the boundary of $X_{0}$. We may define $X_{0}$ canonically, by sweeping out the half-infinite cylinder at $p_{i}$ (or $q_{i}$ ) with lines of slope equal to the residue (considered as element in $\mathbb{R}^{2}$ ) of $\omega$ at $p_{i}$ until such a line hits a zero of $\omega$, ie a singularity of the flat structure.

With this normalization, the above discussion shows that for irreducible stable curves the hyperelliptic involution exchanges the half-infinite cylinders corresponding to $p_{i}$ and $q_{i}$ and it defines an involution $\phi$ of $X_{0}$. As in the smooth case, $\phi$ acts as -Id on $X_{0}$.

To obtain smooth fibers over the Teichmüller curve (in a neighborhood of $X_{\infty}$ ) one has to glue cylinders of finite (large) height in place of the half-infinite cylinders of appropriate ratios of moduli. The hypothesis on $\phi$ acting on $X_{0}$ and on the half-infinite cylinders implies that $\phi$ is a well-defined involution on the smooth curves. Moreover, $\phi$ has two fixed points in each of the finite cylinders and $2 h+2$ fixed points on $X_{0}$, making $2 g+2$ fixed points in total. This shows that the smooth fibers of the Teichmüller curve are hyperelliptic.

To complete the proof we have to consider the two-component degenerations for $\mu \in\{(3,1),(5,1),(5,3)\}$ by Corollary 3.3 . In all these cases, the hyperelliptic involutions can neither exchange the components (since the zeros are of different order) nor fix the components (since the zeros are of odd order).

For $\mu=(3,3)$ a hyperelliptic involution $\phi$ cannot fix the component, since 3 is odd. It cannot exchange the two components and exchange a pair of half-infinite cylinders that belong to different nodes, since $\phi$ could then be used to define a nontrivial involution for each component. This involution fixes the zeros and this contradicts that 3 is odd. If $\phi$ exchanges all pairs of half-infinite cylinders that belong to the same node, $\phi$ has two fixed points in each cylinder on the smooth 'opened up' surface. Now we can apply the same argument as in the irreducible case to conclude that the 'opened up' flat surfaces are hyperelliptic as well.

For $\mu=(3,2,1)$ a hyperelliptic involution can neither fix the component with the (unique) zero of order three, since 3 is odd, nor map it elsewhere, since the zeros are of different order. 


\section{Lyapunov exponents, Siegel-Veech constants and slopes}

\subsection{Lyapunov exponents}

Fix an $\mathrm{SL}_{2}(\mathbb{R})$-invariant, ergodic measure $m$ on $\Omega \mathcal{M}_{g}$. The Lyapunov exponents for the Teichmüller geodesic flow on $\Omega \mathcal{M}_{g}$ measure the logarithm of the growth rate of the Hodge norm of cohomology classes during parallel transport along the geodesic flow. More precisely, let $V$ be the restriction of the real Hodge bundle (ie the bundle with fibers $\left.H^{1}(X, \mathbb{R})\right)$ to the support $M$ of $m$. Let $S_{t}$ be the lift of the geodesic flow to $V$ via the Gauss-Manin connection. Then Oseledec's theorem shows the existence of a filtration

$$
V=V_{\lambda_{1}} \supset \cdots \supset V_{\lambda_{k}} \supset 0
$$

by measurable vector subbundles with the property that, for almost all $p \in M$ and all $v \in V_{p} \backslash\{0\}$, one has

$$
\left\|S_{t}(v)\right\|=\exp \left(\lambda_{i} t+o(t)\right)
$$

where $i$ is the maximum value such that $v$ is in the fiber of $V_{i}$ over $p$, ie $v \in\left(V_{i}\right)_{p}$. The numbers $\lambda_{i}$ for $i=1, \ldots, k \leq \operatorname{rank}(V)$ are called the Lyapunov exponents of $S_{t}$. Note that these exponents are unchanged if we replace the support of $m$ by a finite unramified covering with a lift of the flow and the pullback of $V$. We adopt the convention to repeat the exponents according to the rank of $V_{i} / V_{i+1}$ such that we will always have $2 g$ of them, possibly some of them equal. Since $V$ is symplectic, the spectrum is symmetric, ie $\lambda_{g+k}=-\lambda_{g-k+1}$. The reader may consult Forni [22] or Zorich [42] for a more detailed introduction to this subject.

Most of our results will be about the sum of Lyapunov exponents, defined as

$$
L=\sum_{i=1}^{g} \lambda_{i} .
$$

This sum depends, of course, on the measure $m$ chosen and we occasionally write $L(m)$ to emphasize this dependence. In particular, one defines Lyapunov exponents for an $\mathrm{SL}_{2}(\mathbb{R})$-invariant suborbifold of $\Omega \mathcal{M}_{g}$ carrying such a measure $m$. We will focus on the case of a Teichmüller curve $C$. Consequently, we use $L(C)$ to denote the sum of its Lyapunov exponents.

The bridge between the 'dynamical' definition of Lyapunov exponents and the 'algebraic' method applied in the sequel is given by the following result. Note that if the VHS splits into direct summands one can apply Oseledec's theorem to the summands individually. The full set of Lyapunov exponents is the union (with multiplicity) of the Lyapunov exponents of the summands. 
Theorem 4.1 (Kontsevich [31], Kontsevich and Zorich [32], Bouw and the second author [5]) If the VHS over the Teichmüller curve contains a sub-VHS $\mathbb{W}$ of rank $2 k$, then the sum of the $k$ corresponding nonnegative Lyapunov exponents equals

$$
\sum_{i=1}^{k} \lambda_{i}^{\mathbb{W}}=\frac{2 \operatorname{deg} \mathbb{W}^{(1,0)}}{2 g(\bar{B})-2+|\Delta|},
$$

where $\mathbb{W}^{(1,0)}$ is the $(1,0)$-part of the Hodge-filtration of the vector bundle associated with $\mathbb{W}$. In particular, we have

$$
\sum_{i=1}^{g} \lambda_{i}=\frac{2 \operatorname{deg} f_{*} \omega_{\overline{\mathcal{X}} / \bar{B}}}{2 g(\bar{B})-2+|\Delta|} .
$$

\subsection{Lyapunov exponents for loci of hyperelliptic flat surfaces}

We recall a result of [15, Section 2.3] that deals with the sum of Lyapunov exponents for Teichmüller curves generated by hyperelliptic curves, more generally for any invariant measure on loci of hyperelliptic flat surfaces. It implies immediately that hyperelliptic strata are nonvarying.

Theorem 4.2 [15] Suppose that $M$ is a regular $\mathrm{SL}_{2}(\mathbb{R})$-invariant suborbifold in a locus of hyperelliptic flat surfaces of some stratum $\Omega \mathcal{M}_{g}\left(m_{1}, \ldots, m_{k}\right)$. Denote by $\left(d_{1}, \ldots, d_{s}\right)$ the orders of singularities of the underlying quadratic differentials on the quotient projective line.

Then the sum of Lyapunov exponents for $M$ is

$$
L(M)=\frac{1}{4} \cdot \sum_{\substack{j \text { such that } \\ d_{j} \text { is odd }}} \frac{1}{d_{j}+2},
$$

where, as usual, we associate the order $d_{i}=-1$ to simple poles.

Corollary 4.3 Hyperelliptic strata are nonvarying. For a Teichmüller curve $C$ generated by $(X, \omega)$ we have

$$
\begin{aligned}
& L(C)=\frac{g^{2}}{2 g-1}, \quad s(C)=8+\frac{4}{g}, \quad \text { if }(X, \omega) \in \Omega \mathcal{M}_{g}^{\text {hyp }}(2 g-2), \\
& L(C)=\frac{g+1}{2}, \quad s(C)=8+\frac{4}{g}, \quad \text { if }(X, \omega) \in \Omega \mathcal{M}_{g}^{\text {hyp }}(g-1, g-1) .
\end{aligned}
$$




\subsection{Siegel-Veech constants, slopes and the sum of Lyapunov exponents}

Write $\mu=\left(m_{1}, \ldots, m_{k}\right)$ for a partition of $2 g-2$. Let $c_{\mu}$ denote the (area) SiegelVeech constant of (the connected component of) the stratum $\Omega \mathcal{M}_{g}(\mu)$. Roughly speaking, $c_{\mu}$ measures the growth rate of the weighted sum of cylinders of length at most $T$ on a flat surface $(X, \omega)$ in $\Omega \mathcal{M}_{g}(\mu)$. The weight for each horizontal cylinder is given by its height/length. Similarly, one can define the Siegel-Veech constant $c(C)$ for a Teichmüller curve $C$, or more generally for any $\mathrm{SL}_{2}(\mathbb{R})$-invariant suborbifold in $\Omega \mathcal{M}_{g}(\mu)$ (see [15; 16] for a comprehensive introduction to Siegel-Veech constants).

Let $\kappa_{\mu}$ be a constant

$$
\kappa_{\mu}=\frac{1}{12} \sum_{i=1}^{k} \frac{m_{i}\left(m_{i}+2\right)}{m_{i}+1},
$$

determined by the signature of the stratum. The Siegel-Veech constant and the sum of Lyapunov exponents are related as follows. The condition of regularity in the next theorem is a technical notion that holds for all known examples of invariant suborbifolds and is expected to hold generally (see [15, Section 1.5] for more details).

Theorem 4.4 [15] For any regular $\mathrm{SL}_{2}(\mathbb{R})$-invariant finite measure $m$ on the stratum $\Omega \mathcal{M}_{g}(\mu)$ we have

$$
L(m)=\kappa_{\mu}+c(m) .
$$

In particular the regularity and hence the equality hold for the measure with support equal to a connected component of $\Omega \mathcal{M}_{g}(\mu)$ and for the measure supported on a Teichmüller curve.

For any given Teichmüller curve at a time this theorem allows to calculate the sum of Lyapunov exponents. It suffices to calculate the cusps (in practice, eg for a squaretiled surface, this amounts to calculating the Veech group) and to evaluate the (area) Siegel-Veech contribution of the cusp.

Let $s(C)$ be the slope of a Teichmüller curve $C$ defined by

$$
s(C)=\frac{\bar{C} \cdot \delta_{0}}{\bar{C} \cdot \lambda} .
$$

Since a Teichmüller curve generated by a flat surface does not intersect $\delta_{i}$ in $\overline{\mathcal{M}}_{g}$ for $i>0$ (see Corollary 3.2), its slope can also be defined as

$$
s(C)=\frac{\bar{C} \cdot \delta}{\bar{C} \cdot \lambda},
$$


where $\delta=\sum_{i=0}^{[g / 2]} \delta_{i}$ is the total boundary divisor. The latter is more commonly used for the slope of an arbitrary one-dimensional family of stable genus $g$ curves.

Given a Teichmüller curve $C$, one has to understand only one of the quantities $L(C)$, $c(C)$ and $s(C)$, because of the relation in Theorem 4.4 and another relation as follows.

Proposition 4.5 For a Teichmüller curve $C$ generated by a flat surface, we have

$$
s(C)=\frac{12 c(C)}{L(C)}=12-\frac{12 \kappa_{\mu}}{L(C)} .
$$

Proof This is a consequence of the Noether formula

$$
12 \lambda=\delta+f_{*}\left(c_{1}^{2}\left(\omega_{\overline{\mathcal{X}} / \bar{C}}\right)\right),
$$

as shown by the first author in [8, Theorem 1.8].

We can also directly see how it works. By Theorem 4.1 we know $\chi \cdot L(C)=2 \operatorname{deg} \lambda$, where $\chi=2 g(C)-2+|\Delta|$. Using the Noether formula, the class of $\omega_{\overline{\mathcal{X}} / \bar{C}}$ in the proof of Proposition 4.8 and Theorem 4.4, we can derive that $6 \chi \cdot c(C)=\operatorname{deg} \delta$. Hence the equality (14) follows immediately.

Now our strategy becomes clear. In order to show a stratum is nonvarying, it suffices to show all Teichmüller curves in that stratum are disjoint from an effective divisor on $\overline{\mathcal{M}}_{g}$, hence they all have the same slope as that of the divisor. Then by (14), they have the same sum of Lyapunov exponents as well. We summarize this idea as follows.

Lemma 4.6 Let $D$ be an effective divisor on $\overline{\mathcal{M}}_{g}$. Suppose the closures of all Teichmüller curves $\bar{C}$ generated by flat surfaces in a fixed stratum do not intersect $D$. Then they have the same slope $s(C)=s(D)$. In particular, the sums of Lyapunov exponents are the same for these Teichmüller curves.

Proof Recall the slope of an effective divisor defined in Section 2.3. Suppose $D$ has class $a \lambda-\sum_{i=0}^{[g / 2]} b_{i} \delta_{i}$. Then it has slope $s(D)=a / b_{0}$. Since $\bar{C} \cdot D=0$ and $\bar{C} \cdot \delta_{i}=0$ for $i>0$ (Corollary 3.2), we conclude that

$$
\frac{\bar{C} \cdot \delta_{0}}{\bar{C} \cdot \lambda}=\frac{a}{b_{0}}
$$

hence $s(C)=s(D)$. Since the slopes are nonvarying, so are the sums of Lyapunov exponents and the Siegel-Veech constants for those Teichmüller curves, according to (14). 
The same argument can help us find upper bounds for the slope as well as for the sum of Lyapunov exponents for a Teichmüller curve.

Lemma 4.7 Let $D$ be an effective divisor on $\overline{\mathcal{M}}_{g}$. Suppose a Teichmüller curve $C$ is not contained in $D$. Then we have $s(C) \leq s(D)$.

Proof Suppose $D$ has class $a \lambda-\sum_{i=0}^{[g / 2]} b_{i} \delta_{i}$. By assumption we have $C \cdot D \geq 0$. It implies that $a(\bar{C} \cdot \lambda)-b_{0}\left(\bar{C} \cdot \delta_{0}\right) \geq 0$, hence

$$
s(C)=\frac{\bar{C} \cdot \delta_{0}}{\bar{C} \cdot \lambda} \leq \frac{a}{b_{0}}=s(D) .
$$

In some cases it is not possible to find an effective divisor on $\overline{\mathcal{M}}_{g}$ to perform the disjointness argument (see eg the explanation in Section 5.3). Alternatively, we have to consider moduli spaces of curves with marked points or spin structures. Consequently we need to know the intersection of Teichmüller curves with the classes $\omega_{i \text {,rel }}$ introduced in Section 2.3.

Let $C$ be a Teichmüller curve generated by $(X, \omega) \in \Omega \mathcal{M}_{g}\left(m_{1}, \ldots, m_{k}\right)$. Let $B \rightarrow C$ be a finite unramified cover such that the $m_{i}$-fold zero defines a section $\sigma_{i}$ (not only a multisection) with image $S_{i}$ of the pullback family $f: \mathcal{X} \rightarrow \bar{B}$.

Proposition 4.8 If $f: \bar{B} \rightarrow \overline{\mathcal{M}}_{g, 1}$ is the lift of a Teichmüller curve by marking the zero of order $m_{i}$, then

$$
S_{i}^{2}=\frac{-\chi}{2\left(m_{i}+1\right)}
$$

where $\chi=2 g(\bar{B})-2+|\Delta|$ and $\Delta$ is the set of cusps in $\bar{B}$. In particular the intersection number with $\omega_{i, \text { rel }}$, which is by definition equal to $-S_{i}^{2}$, is given by

$$
\bar{B} \cdot \omega_{i, \text { rel }}=\frac{\bar{B} \cdot \lambda-(\bar{B} \cdot \delta) / 12}{\left(m_{i}+1\right) \kappa_{\mu}},
$$

where $\kappa_{\mu}=\frac{1}{12} \sum_{j=1}^{k} \frac{m_{j}\left(m_{j}+2\right)}{m_{j}+1}$.

Proof Let $\mathcal{L} \subset f_{*} \omega_{\overline{\mathcal{X}} / \bar{B}}$ be the ('maximal Higgs', see [36]) line bundle whose fiber over the point corresponding to $[X]$ is $\mathbb{C} \cdot \omega$, the generating differential of the Teichmüller curve. The property 'maximal Higgs' says by definition that

$$
\operatorname{deg}(\mathcal{L})=\chi / 2 \text {. }
$$


Let $S$ be the union of the sections $S_{1}, \ldots, S_{k}$. Pulling back the above inclusion to $\mathcal{X}$ gives an exact sequence

$$
0 \rightarrow f^{*} \mathcal{L} \rightarrow \omega_{\overline{\mathcal{X}} / \bar{B}} \rightarrow \mathcal{O}_{S}\left(\sum_{j=1}^{k} m_{j} S_{j}\right) \rightarrow 0,
$$

since the multiplicities of the vanishing locus of the generating differential of the Teichmüller curve are constant along the whole compactified Teichmüller curve. This implies that $\omega_{\mathcal{X} / \bar{B}}$ is numerically equal to

$$
f^{*} \mathcal{L}+\sum_{j=1}^{k} m_{j} S_{j}
$$

By the adjunction formula we get

$$
S_{i}^{2}=-\omega_{\overline{\mathcal{X}} / \bar{B}} \cdot S_{i}=-m_{i} S_{i}^{2}-\operatorname{deg}(\mathcal{L}),
$$

since the intersection product of two fibers of $f$ is zero. Together with (15) we thus obtain the desired self-intersection formula.

By Theorem 4.1 and the relation (14), we have

$$
\begin{gathered}
\bar{B} \cdot \lambda=\frac{\chi}{2} \cdot L, \\
\bar{B} \cdot \delta=\frac{\chi}{2} \cdot\left(12 L-12 \kappa_{\mu}\right) .
\end{gathered}
$$

Hence the second claimed formula follows, for $-S_{i}^{2}=\bar{B} \cdot \omega_{i, \text { rel }}$ by definition.

Remark 4.9 For square-tiled surfaces the self-intersection number of a section on the elliptic surface is not hard to calculate (see Kodaira [30]), recalled by the second author in [37], and also by the first author in [7, Theorem 1.15]). Pullback introduces the coefficient $m_{i}+1$ in the denominator. This shows the formula in the square-tiled case. The general case of the formula can also be shown by adapting the argument given by Bainbridge in [3, Theorem 12.2], since there are $m_{i}+1$ ways to split a singularity of order $m_{i}$.

If $L$ is nonvarying for all Teichmüller curves (or just those generated by square-tiled surfaces) in a stratum $\Omega \mathcal{M}_{g}(\mu)$, it implies that the sum of Lyapunov exponents for the whole stratum is equal to $L$.

Proposition 4.10 As the area (ie the degree of the torus coverings) approaches infinity, the limit of sums of Lyapunov exponents for Teichmüller curves generated by squaretiled surfaces in a stratum is equal to the sum of Lyapunov exponents for that stratum. 
This is due to the fact that square-tiled surfaces in a stratum are parameterized by 'lattice points' under the period coordinates (see eg Eskin and Okounkov [17, Lemma 3.1]). Hence, their asymptotic behavior reveals information for the whole stratum (see eg [8, Appendix A] for a proof).

\section{Genus three}

In genus 3 all the strata have nonvarying sums of Lyapunov exponents except the principal stratum. We summarize the results in Figure 2. We also give a sharp upper bound for the sum of Lyapunov exponents for the principal stratum.

Let us first explain how to read the table. For example, the stratum $(2,2)^{\text {odd }}$ is nonvarying. The sum of Lyapunov exponents is equal to $\frac{5}{3}(\approx 1.66666)$ for both the stratum and every Teichmüller curve in the stratum. On the other hand, the principal stratum $(1,1,1,1)$ is varying. The sum of Lyapunov exponents for the whole stratum is $\frac{53}{28}(\approx 1.89285)$. The sharp upper bound for the sums of Lyapunov exponents of Teichmüller curves in this stratum is 2 . It can be attained, eg by Teichmüller curves in the locus of hyperelliptic flat surfaces as the image of $\mathcal{Q}\left(2,2,-1^{8}\right)$ in the context of Theorem 4.2. Later on we will use similar tables to encode the behavior of Teichmüller curves in other genera.

\subsection{The stratum $\Omega \mathcal{M}_{3}(4)^{\text {odd }}$}

In the case $\Omega \mathcal{M}_{3}(4)^{\text {odd }}$ the algorithm of [16] to calculate Siegel-Veech constants for components of strata gives

$$
L_{(4)}{ }^{\text {odd }}=8 / 5, \quad s_{(4)}{ }^{\text {odd }}=9, \quad c_{(4)} \text { odd }=6 / 5 .
$$

Proof of Theorem 1.1, Case $\Omega \mathcal{M}_{3}(4)^{\text {odd }}$ The connected components $\Omega \mathcal{M}_{3}$ (4) ${ }^{\text {odd }}$ and $\Omega \mathcal{M}_{3}(4)^{\text {hyp }}$ are not only disjoint in $\Omega \mathcal{M}_{3}$, by Proposition 3.4 they are also disjoint in $\Omega \overline{\mathcal{M}}_{3}$. Hence a Teichmüller curve $\bar{C}$ generated by a flat surface in this stratum do not intersect the hyperelliptic locus $H$ in $\overline{\mathcal{M}}_{3}$. Recall the divisor class of $H$ in (1). By Lemma 4.6 and $s(H)=9$, we obtain that $s(C)=9$, hence $c(C)=6 / 5$ and $L(C)=8 / 5$ for all Teichmüller curves in this stratum using (13) and (14).

\subsection{The stratum $\Omega \mathcal{M}_{3}(3,1)$}

In the case $\Omega \mathcal{M}_{3}(3,1)$ we have

$$
L_{(3,1)}=7 / 4, \quad s_{(3,1)}=9, \quad c_{(3,1)}=21 / 16 .
$$




\begin{tabular}{|c|c|c|c|c|c|c|}
\hline \multirow{3}{*}{$\begin{array}{c}\text { Degrees } \\
\text { of } \\
\text { zeros } \\
\left(d_{1}, \ldots, d_{n}\right)\end{array}$} & \multirow{3}{*}{$\begin{array}{c}\text { Hyperelliptic } \\
\text { or spin } \\
\text { structure }\end{array}$} & \multicolumn{5}{|c|}{ Lyapunov exponents } \\
\hline & & \multicolumn{2}{|c|}{ Component } & \multicolumn{3}{|c|}{ Teichmüller curves } \\
\hline & & $\approx$ & $\sum_{j=1}^{g} \lambda_{j}$ & $\approx$ & $\sum_{j=1}^{g} \lambda_{j}$ & Reference \\
\hline (4) & hyperelliptic & 1.80000 & $\frac{9}{5}$ & \multicolumn{2}{|c|}{ Nonvarying } & Theorem 4.2 \\
\hline (4) & odd & 1.60000 & $\frac{8}{5}$ & \multicolumn{2}{|c|}{ Nonvarying } & Section 5.1 \\
\hline$(3,1)$ & - & 1.75000 & $\frac{7}{4}$ & \multicolumn{2}{|c|}{ Nonvarying } & Section 5.2 \\
\hline$(2,2)$ & hyperelliptic & 2.00000 & 2 & \multicolumn{2}{|c|}{ Nonvarying } & Theorem 4.2 \\
\hline$(2,2)$ & odd & 1.66666 & $\frac{5}{3}$ & \multicolumn{2}{|c|}{ Nonvarying } & Section 5.3 \\
\hline$(2,1,1)$ & - & 1.83333 & $\frac{11}{6}$ & \multicolumn{2}{|c|}{ Nonvarying } & Section 5.4 \\
\hline$(1,1,1,1)$ & - & 1.89285 & $\frac{53}{28}$ & 2 & 2 & $\mathcal{Q}\left(2,2,-1^{8}\right)$ \\
\hline
\end{tabular}

Figure 2: Varying and nonvarying sums in genus three

Proof of Theorem 1.1, Case $\Omega \mathcal{M}_{3}(3,1)$ As in the case of the stratum $\Omega \mathcal{M}_{3}(4)^{\text {odd }}$, a Teichmüller curve $C$ generated by a flat surface in the stratum $(3,1)$ does not intersect the hyperelliptic locus, not even at the boundary by Proposition 3.4. Consequently we can apply the same disjointness argument as in the preceding case. Since $s(H)=9$, we obtain that $s(C)=9$, hence $c(C)=21 / 16$ and $L(C)=7 / 4$ using (13) and (14).

\subsection{The stratum $\Omega \mathcal{M}_{3}(2,2)^{\text {odd }}$}

In the case $\Omega \mathcal{M}_{3}(2,2)^{\text {odd }}$, we have

$$
L_{(2,2)^{\text {odd }}}=5 / 3, \quad s_{(2,2)^{\text {odd }}}=44 / 5, \quad c_{(2,2) \text { odd }}=11 / 9 .
$$

Note that on $\overline{\mathcal{M}}_{3}$ the smallest slope of an effective divisor is 9 (attained by the divisor $H$ of hyperelliptic curves; see Harris and Morrison [26, Theorem 0.4]). In order to show a Teichmüller curve $\bar{C}$ has $L(C)=44 / 5$ in this stratum, we cannot use an effective divisor $D$ on $\overline{\mathcal{M}}_{3}$ such that $\bar{C} \cdot D=0$. Otherwise by Lemma 4.6 it would 
imply that $s(C)=s(D) \geq 9>44 / 5$. Instead, we have to use another moduli space parameterizing curves with some additional structure. Here the stratum is distinguished by spin structures, hence it is natural to consider the spin moduli space introduced in Section 2.2.

Since the same idea will also be applied to the stratum $\Omega \mathcal{M}_{4}(2,2,2)^{\text {odd }}$, we first consider the general case $\Omega \mathcal{M}_{g}(2, \ldots, 2)^{\text {odd }}$. Let $C$ be a Teichmüller curve generated by a flat surface $(X, \omega)$ in $\Omega \overline{\mathcal{M}}_{g}(2, \ldots, 2)^{\text {odd }}$ such that $\operatorname{div}(\omega)=2 \sum_{i=1}^{g-1} p_{i}$ for distinct points $p_{i}$. Using $\eta=\sum_{i=1}^{g-1} p_{i}$ as an odd theta characteristic, we can map $\bar{C}$ to $\overline{\mathcal{S}}_{g}^{-}$.

Let $\bar{Z}_{g}$ be the divisor on $\overline{\mathcal{S}}_{g}^{-}$parameterizing $(X, \eta)$ such that the odd theta characteristic $\eta$ satisfies

$$
\eta \sim \mathcal{O}_{X}\left(2 p_{1}+p_{2}+\cdots+p_{g-2}\right) .
$$

The class of $\bar{Z}_{g}$ was calculated in [20, Theorem 0.4]:

$$
\bar{Z}_{g}=(g+8) \lambda-\frac{g+2}{4} \alpha_{0}-2 \beta_{0}-\sum_{i=1}^{[g / 2]} 2(g-i) \alpha_{i}-\sum_{i=1}^{[g / 2]} 2 i \beta_{i},
$$

where $\lambda$ is the pullback of the $\lambda$-class on $\overline{\mathcal{M}}_{g}$ and $\alpha_{i}, \beta_{i}$ are two different boundary divisors of $\overline{\mathcal{S}}_{g}^{-}$over $\delta_{i}$ for each $0 \leq i \leq[g / 2]$. Since Teichmüller curves do not intersect $\delta_{i}$ for $i>0$, we focus on $\delta_{0}$ and its inverse images $\alpha_{0}, \beta_{0}$ only. By definition in [20, Section 1.2], a spin curve $Y$ in $\beta_{0}$ possesses an exceptional component $E$, ie a rational curve that meets the rest of $Y$ at two nodes, and the theta characteristic $\eta$ has degree 1 restricted to $E$. In particular, $Y$ cannot be parameterized in the boundary of a Teichmüller curve, since none of the zeros $p_{i}$ will lie on $E$ due to the fact that $\left.\omega_{Y}\right|_{E}$ has degree 0 and Corollary 3.2. We thus conclude the following.

Lemma 5.1 In the above setting, $\bar{C}$ does not intersect any boundary components of $\overline{\mathcal{S}}_{g}^{-}$except $\alpha_{0}$.

We are interested in the case when an odd theta characteristic does not have extra sections.

Proposition 5.2 In the above setting, suppose every odd theta characteristic $\eta$ parameterized in $\mathcal{S}_{g}^{-}$union $\alpha_{0}$ satisfies $h^{0}(\eta)=1$. Then the slope of $C$ is

$$
s(C)=\frac{4(g+8)}{g+2} .
$$


Proof In $\mathcal{S}_{g}^{-}$union $\alpha_{0}, \bar{Z}_{g}$ can be identified as the stratum $\Omega \overline{\mathcal{M}}_{g}(4,2, \ldots, 2)^{\text {odd }}$, since the unique section of $\eta$ determines the zeros of the corresponding Abelian differential. Consequently by Proposition 3.1, the image of $\bar{C}$ in $\overline{\mathcal{S}}_{g}^{-}$does not intersect $\bar{Z}_{g}$. Then we have

$$
0=\bar{C} \cdot \bar{Z}_{g}=\bar{C} \cdot\left((g+8) \lambda-\frac{g+2}{4} \alpha_{0}\right),
$$

since $\bar{C}$ does not intersect any boundary components except $\alpha_{0}$ by Lemma 5.1. Note that $\pi^{*} \lambda=\lambda$ and $\pi^{*} \delta_{0}=\alpha_{0}+2 \beta_{0}$, where $\pi: \overline{\mathcal{S}}_{g}^{-} \rightarrow \overline{\mathcal{M}}_{g}$ is the morphism forgetting the spin structure and stabilizing the curve (see [20, Section 1.2]). By the projection formula we have

$$
0=\left(\pi_{*} \bar{C}\right) \cdot\left((g+8) \lambda-\frac{g+2}{4} \delta_{0}\right) .
$$

The desired formula follows right away.

We remark that the assumption $h^{0}(\eta)=1$ for all $\eta$ is rather strong and seems to hold only in low genus, as a consequence of Clifford's theorem (Theorem 2.5).

Proof of Theorem 1.1, Case $\Omega \mathcal{M}_{3}(2,2)^{\text {odd }}$ For $g=3$ an odd theta characteristic cannot have three or more sections by Theorem 2.5. Hence Proposition 5.2 applies and we obtain that $s(C)=44 / 5$.

Note that this argument does not distinguish between hyperelliptic and nonhyperelliptic curves and for double covers of $\mathcal{Q}\left(1,1,-1^{6}\right)$ the result is in accordance with Theorem 4.2.

\subsection{The stratum $\Omega \mathcal{M}_{3}(2,1,1)$}

In the case $\Omega \mathcal{M}_{3}(2,1,1)$ we have

$$
L_{(2,1,1)}=11 / 6, \quad s_{(2,1,1)}=98 / 11, \quad c_{(2,1,1)}=49 / 36 .
$$

Since $s_{(2,1,1)}=98 / 11<9$, by the same reason as in the preceding section, one cannot verify this nonvarying slope by disjointness with an effective divisor on $\overline{\mathcal{M}}_{3}$. This time the flat surfaces have three zeros, hence it is natural to consider the moduli space of pointed curves by marking some of the zeros. Consequently we will seek certain pointed Brill-Noether divisors to perform the disjointness argument.

First we lift a Teichmüller curve generated by a flat surface in this stratum to $\overline{\mathcal{M}}_{3,2}$ by marking the double zero $p$ as the first point and one of the simple zeros $q$ as the second point. In fact, we can do so after passing to a double covering of $C$ where the simple zeros $q$ and $r$ can be distinguished. This double covering is unramified, 
since by definition of a Teichmüller curve the zeros never collide. Moreover, the slope and hence the sum of Lyapunov exponents are unchanged by passing to an unramified double covering. For simplicity we will continue to call $C$ the Teichmüller curve we work with.

Proposition 5.3 In the above setup, the Teichmüller curve $\bar{C}$ does not intersect the pointed Brill-Noether divisor $B N_{3,(1,2)}^{1}$ on $\overline{\mathcal{M}}_{3,2}$.

Proof Recall that $B N_{3,(1,2)}^{1}$ parameterizes pointed curves $(X, p, q)$ that possess a $g_{3}^{1}$ containing $p+2 q$ as a section. Suppose $(X, \omega)$ is in the intersection of $\bar{C}$ and $B N_{3,(1,2)}^{1}$. Since $h^{0}\left(\mathcal{O}_{X}(p+2 q)\right)=2$ and $p, q, r$ are distinct, we obtain that $h^{0}\left(\mathcal{O}_{X}(p+r-q)\right)=1$ by Riemann-Roch and then $h^{0}\left(\mathcal{O}_{X}(p+r)\right)=2$. If $X$ is smooth, then $X$ is hyperelliptic and $p, r$ are conjugate. But $\omega_{X} \sim \mathcal{O}_{X}(2 p+q+r)$, so $p, q$ are also conjugate, contradiction. For singular $X$ we deduce from $h^{0}\left(\mathcal{O}_{X}(p+r)\right)=2$ that $p$ and $r$ are in the same component $X_{0}$ of $X$. This component admits an involution $\phi$ that acts on the set of zeros of $\left.\omega_{X}\right|_{X_{0}}$. But $p$ and $r$ have different orders, so they cannot be conjugate under $\phi$, leading to a contradiction.

Proof of Theorem 1.1, Case $\Omega \mathcal{M}_{3}(2,1,1)$ By the proposition and the divisor class of $B N_{3,(1,2)}^{1}$ in (5), we obtain that

$$
\bar{C} \cdot\left(-\lambda+\omega_{1, \text { rel }}+3 \omega_{2, \text { rel }}\right)=0 .
$$

Since $\kappa_{(2,1,1)}=17 / 36$, using Proposition 4.8, we have

$$
\begin{aligned}
& \bar{C} \cdot \omega_{1, \text { rel }}=\frac{\bar{C} \cdot \lambda-(\bar{C} \cdot \delta) / 12}{17 / 12}, \\
& \bar{C} \cdot \omega_{2, \text { rel }}=\frac{\bar{C} \cdot \lambda-(\bar{C} \cdot \delta) / 12}{17 / 18} .
\end{aligned}
$$

Plugging in the above, we obtain that $s(C)=98 / 11$ and the values of $L(C), c(C)$ follow from (13) and (14).

Remark 5.4 Alternatively, the theorem can be deduced by showing that for a Teichmüller curve $\bar{C}$ its intersection loci with the hyperelliptic locus $H$ in $\overline{\mathcal{M}}_{3}$ and with the Weierstrass divisor $W$ in $\overline{\mathcal{M}}_{3,1}$ are the same. We show that these intersections are set-theoretically equal. A complete proof via this method would need to verify that the intersection multiplicities with the two divisors coincide.

Hyperelliptic flat surfaces in this stratum are obtained as coverings from the stratum $\mathcal{Q}\left(2,1,-1^{7}\right)$ and we deduce from Theorem 4.2 that $L_{(2,1,1)}^{\text {hyp }}=11 / 6$. Hence we may 
assume that $\bar{C}$ is not entirely in the hyperelliptic locus. If $\bar{C}$ intersects $W$ at a point $(X, 2 p, q, r)$, where $p$ is the marked point, then $p$ is a Weierstrass point. Hence we have $2 p+q+r \sim 3 p+s$ for some $s$ in $X$. Consequently $q+r \sim p+s$ and $X$ must be hyperelliptic. On the other hand, suppose $\bar{C}$ intersects $H$ at a point $(X, 2 p, q, r)$. Since $X$ is hyperelliptic, $p$ must be a Weierstrass point.

Corollary 5.5 A Teichmüller curve generated by a nonhyperelliptic flat surface in $\Omega \mathcal{M}_{3}(2,1,1)$ does intersect the hyperelliptic locus $H$ at the boundary.

Proof If the statement was false for some Teichmüller curve $C$, we would have $\bar{C} \cdot H=0$, hence $s(C)=s(H)=9$, contradicting $s(C)=98 / 11$.

\subsection{Varying sum in the stratum $\Omega \mathcal{M}_{3}(1,1,1,1)$}

We show by example that the sum of Lyapunov exponents in the principal stratum in $g=3$ is varying, even modulo the hyperelliptic locus. In the case $\Omega \mathcal{M}_{3}(1,1,1,1)$, the algorithm of [16] to calculate Siegel-Veech constants for components of strata gives

$$
L_{(1,1,1,1)}=53 / 28, \quad s_{(1,1,1,1)}=468 / 53, \quad c_{(1,1,1,1)}=39 / 28 .
$$

Examples The 'eierlegende Wollmilchsau', the square-tiled surface given by the permutations $\left(\pi_{r}=(1234)(5678), \pi_{u}=(1836)(2745)\right)$ (see [22; 37]), generates a Teichmüller curve $C$ with $L(C)=1$.

The square-tiled surface given by the permutations

$$
\left(\pi_{r}=(1234)(5)(6789), \pi_{u}=(1)(2563)(4897)\right)
$$

generates a Teichmüller curve $C$ with $L(C)=2$. It attains the upper bound given by Theorem 1.1, but it is not hyperelliptic.

There exist square-tiled surfaces in this stratum whose associated Teichmüller curves $C$ have

$$
L(C) \in\{1,3 / 2,5 / 3,7 / 4,9 / 5,11 / 6,19 / 11,33 / 19,83 / 46,544 / 297\} .
$$

Proof of Theorem 1.1, Case $\Omega \mathcal{M}_{3}(1,1,1,1)$ Teichmüller curves in the locus of hyperelliptic flat surfaces (ie the image of $\mathcal{Q}\left(2,2,-1^{8}\right)$ ) in $\Omega_{\mathcal{M}}(1,1,1,1)$ have $L=2$ by Theorem 4.2 .

If the Teichmüller curve $C$ is not contained in the hyperelliptic locus, then $\bar{C} \cdot H \geq 0$, or equivalently $s(C) \leq s(H)=9$ by Lemma 4.7. Using $\kappa_{(1,1,1,1)}=1 / 2$ this implies $L(C) \leq 2$.

For the last statement in the theorem recall that an double cover of a genus two curve is always hyperelliptic (eg Farkas [21]). 


\section{Genus four}

In genus 4 we summarize the nonvarying sums of Lyapunov exponents and upper bounds for varying sums in Figure 3.

\subsection{The stratum $\Omega \mathcal{M}_{4}(6)^{\text {even }}$}

In the case $\Omega \mathcal{M}_{4}(6)^{\text {even }}$, we have

$$
\left.L_{(6)}\right)^{\text {even }}=14 / 7, \quad s_{(6)^{\text {even }}}=60 / 7, \quad c_{(6)^{\text {even }}}=10 / 7 .
$$

Proposition 6.1 Let $C$ be a Teichmüller curve generated by $(X, \omega) \in \Omega \mathcal{M}_{4}(6)^{\text {even }}$, lifted to $\overline{\mathcal{M}}_{4,1}$ using the zero of $\omega$. Then $\bar{C}$ does not intersect the theta-null divisor $\Theta$ in $\overline{\mathcal{M}}_{4,1}$.

Proof Recall that the divisor $\Theta \subset \overline{\mathcal{M}}_{4,1}$ parameterizes curves that admit an odd theta characteristic whose support contains the marked point. Suppose the stable pointed curve $(X, p)$ lies in the intersection of $\bar{C}$ and $\Theta$. Then there exists an odd theta characteristic $\eta$ on $X$ with a section $t \in H^{0}(\eta)$ such that $\operatorname{div}(t)=p+q+r$ for some $q, r$ not both equal to $p$. Denote by $\mathcal{L}=\mathcal{O}_{X}(3 p)$ the line bundle corresponding to the even theta characteristic with a section $s \in H^{0}(\mathcal{L})$ given by $3 p$. Since $\eta^{\otimes 2} \sim \omega_{X} \sim \mathcal{L}^{\otimes 2}$, the function $s^{2} t^{-2}$ implies that $4 p \sim 2 q+2 r$ on $X$, hence $p$ is not a base point of $|\mathcal{L}(p)|=\left|\mathcal{O}_{X}(4 p)\right|$. Consequently we have $h^{0}(\mathcal{L}(p))=1+h^{0}(\mathcal{L}) \geq 3$. By $\omega_{X} \sim \mathcal{O}_{X}(6 p)$ and Riemann-Roch, $h^{0}\left(\mathcal{O}_{X}(2 p)\right)=h^{0}\left(\mathcal{O}_{X}(4 p)\right)-1 \geq 2$. Since $X$ is irreducible by Corollary 3.2, this implies that $X$ is hyperelliptic and $p$ is a Weierstrass point. It contradicts the disjointness of the hyperelliptic locus and this component in $\overline{\mathcal{M}}_{4}$ by Proposition 3.4 .

Proof of Theorem 1.2, Case $\Omega \mathcal{M}_{4}(6)^{\text {even }}$ Using the proposition and the class of the theta-null divisor $\Theta$ in (3), we obtain that

$$
\bar{C} \cdot\left(30 \lambda+60 \omega_{\text {rel }}-4 \delta_{0}\right)=0 .
$$

Using Proposition 4.8 we know

$$
\bar{C} \cdot \omega_{\text {rel }}=\frac{\bar{C} \cdot \lambda-(\bar{C} \cdot \delta) / 12}{4} .
$$

It now suffices to plug this in and use (13) and (14). 


\begin{tabular}{|c|c|c|c|c|c|c|}
\hline \multirow{3}{*}{$\begin{array}{c}\text { Degrees } \\
\text { of } \\
\text { zeros } \\
\left(d_{1}, \ldots, d_{n}\right)\end{array}$} & \multirow{3}{*}{$\begin{array}{c}\text { Hyperelliptic } \\
\text { or spin } \\
\text { structure }\end{array}$} & \multicolumn{5}{|c|}{ Lyapunov exponents } \\
\hline & & \multicolumn{2}{|c|}{ Component } & \multicolumn{3}{|c|}{ Teichmüller curves } \\
\hline & & $\approx$ & $\sum_{j=1}^{g} \lambda_{j}$ & $\approx$ & $\sum_{j=1}^{g} \lambda_{j}$ & Reference \\
\hline (6) & hyperelliptic & 2.28571 & $\frac{16}{7}$ & \multicolumn{2}{|c|}{ Nonvarying } & Theorem 4.2 \\
\hline (6) & even & 2.00000 & 2 & \multicolumn{2}{|c|}{ Nonvarying } & Section 6.1 \\
\hline (6) & odd & 1.85714 & $\frac{13}{7}$ & \multicolumn{2}{|c|}{ Nonvarying } & Section 6.2 \\
\hline$(5,1)$ & - & 2.00000 & 2 & \multicolumn{2}{|c|}{ Nonvarying } & Section 6.3 \\
\hline$(4,2)$ & even & 2.13333 & $\frac{32}{15}$ & \multicolumn{2}{|c|}{ Nonvarying? } & - \\
\hline$(4,2)$ & odd & 1.93333 & $\frac{29}{15}$ & \multicolumn{2}{|c|}{ Nonvarying? } & - \\
\hline$(3,3)$ & hyperelliptic & 2.50000 & $\frac{5}{2}$ & \multicolumn{2}{|c|}{ Nonvarying } & Theorem 4.2 \\
\hline$(3,3)$ & non - hyp & 2.00000 & 2 & \multicolumn{2}{|c|}{ Nonvarying } & Section 6.6 \\
\hline$(3,2,1)$ & - & 2.08333 & $\frac{25}{12}$ & \multicolumn{2}{|c|}{ Nonvarying } & Section 6.8 \\
\hline$(2,2,2)$ & odd & 2.00000 & 2 & \multicolumn{2}{|c|}{ Nonvarying } & Section 6.7 \\
\hline$(2,2,2)$ & even & 2.28571 & $\frac{166}{75}$ & 2.333333 & $\frac{7}{3}$ & $\mathcal{Q}\left(3,1,-1^{8}\right)$ \\
\hline$(4,1,1)$ & - & 2.06727 & $\frac{1137}{550}$ & 1.96792 & $\frac{1043}{530}$ & (16) \\
\hline$\left(2^{2}, 1^{2}\right)$ & - & 2.13952 & $\frac{5045}{2358}$ & 1.91666 & $\frac{23}{12}$ & $\mathcal{Q}\left(2,1,1,-1^{7}\right)$ \\
\hline$\left(3,1^{3}\right)$ & - & 2.12903 & $\frac{66}{31}$ & 2.11523 & $\frac{514}{243}$ & (17) \\
\hline$\left(2,1^{4}\right)$ & - & 2.18333 & $\frac{131}{60}$ & 2.80000 & $\frac{14}{5}$ & $\mathcal{Q}\left(3,2,2,-1^{11}\right)$ \\
\hline$\left(1^{6}\right)$ & - & 2.22546 & $\frac{839}{377}$ & 2.50000 & $\frac{5}{2}$ & $\mathcal{Q}\left(2,2,2,-1^{10}\right)$ \\
\hline
\end{tabular}

Figure 3: Varying and nonvarying sums in genus four 


\subsection{The stratum $\Omega \mathcal{M}_{4}(6)^{\text {odd }}$}

In the case $\Omega \mathcal{M}_{4}(6)^{\text {odd }}$, we have

$$
L_{(6)^{\text {odd }}}=13 / 7, \quad s_{(6)^{\text {odd }}}=108 / 13, \quad c_{(6)^{\text {odd }}}=9 / 7 .
$$

Proposition 6.2 Let $C$ be a Teichmüller curve generated by $(X, \omega) \in \Omega_{4}(6)^{\text {odd }}$ lifted to $\overline{\mathcal{M}}_{4,1}$ using the zero of $\omega$. Then $C$ does not intersect the pointed Brill-Noether divisor $B N_{3,(2)}^{1}$.

Proof Recall that $B N_{3,(2)}^{1} \subset \overline{\mathcal{M}}_{4,1}$ parameterizes curves that admit a linear series $g_{3}^{1}$ with a section containing $2 p$, where $p$ is the marked point. Suppose $\bar{C}$ intersects $B N_{3,(2)}^{1}$ at $(X, p)$. Let $\eta=\mathcal{O}_{X}(3 p)$ denote the theta characteristic given by $3 p$. Since $h^{0}(\eta)$ is odd, Clifford's theorem implies that $h^{0}(\eta)=1$. Since $(X, p)$ is contained in $B N_{3,(2)}^{1}$, we have $h^{0}\left(\mathcal{O}_{X}(2 p+q)\right)=2$ for some $q$ different from $p$. By $\omega_{X} \sim \mathcal{O}_{X}(6 p)$ and Riemann-Roch, we have $h^{0}\left(\mathcal{O}_{X}(4 p-q)\right)=2$, hence $h^{0}\left(\mathcal{O}_{X}(3 p-q)\right) \geq 1$. Note that $q$ is not a base point of the linear system $\left|\mathcal{O}_{X}(3 p)\right|$ for $q \neq p$. Consequently we have $h^{0}(\eta)=1+h^{0}\left(\mathcal{O}_{X}(3 p-q)\right) \geq 2$, contradicting that $h^{0}(\eta)=1$.

Proof of Theorem 1.2, Case $\Omega \mathcal{M}_{4}(6)^{\text {odd }} \quad$ Recall the divisor class of $B N_{3,(2)}^{1}$ in (7). By $\bar{C} \cdot B N_{3,(2)}^{1}=0$, we have

$$
\bar{C} \cdot\left(4 \omega_{\text {rel }}+8 \lambda-\delta_{0}\right)=0 .
$$

It now suffices to use Proposition 4.8 and to plug the result in (13) and (14).

\subsection{The stratum $\Omega \mathcal{M}_{4}(5,1)$}

In the case $\Omega \mathcal{M}_{4}(5,1)$, we have

$$
L_{(5,1)}=2, \quad s_{(5,1)}=25 / 3, \quad c_{(5,1)}=25 / 18 .
$$

Proposition 6.3 Let $C$ be a Teichmüller curve generated by $(X, \omega) \in \Omega \mathcal{M}_{4}(5,1)$, lifted to $\overline{\mathcal{M}}_{4,1}$ using the 5-fold zero of $\omega$. Then $\bar{C}$ does not intersect the pointed Brill-Noether divisor $B N_{3,(2)}^{1}$.

Proof Suppose that $(X, \omega)$ is contained in the intersection of $\bar{C}$ with $B N_{3,(2)}^{1}$, where $\operatorname{div}(\omega)=5 p+q$ with $p$ the marked point. By Proposition 3.4, $X$ is not hyperelliptic. For a nonhyperelliptic curve $X$ which is either smooth, or nodal irreducible, or consisting of two components joined at three nodes, its dual graph is three-connected, 
hence the dualizing sheaf $\omega_{X}$ is very ample due to Proposition 2.6. We will analyze the geometry of its canonical image in $\mathbb{P}^{3}$.

We have $h^{0}\left(\mathcal{O}_{X}(2 p+r)\right) \geq 2$ for some smooth point $r$. Since $\omega_{X} \sim \mathcal{O}_{X}(5 p+q)$, by Riemann-Roch, it implies that $h^{0}\left(\mathcal{O}_{X}(3 p+q-r)\right) \geq 2$, hence $h^{0}\left(\mathcal{O}_{X}(3 p-r)\right) \geq 1$. If $r \neq p$, then $r$ is not a base point of $\left|\mathcal{O}_{X}(3 p)\right|$, hence $h^{0}\left(\mathcal{O}_{X}(3 p)\right) \geq 2$. If $r=p$, then we still have $h^{0}\left(\mathcal{O}_{X}(3 p)\right)=h^{0}\left(\mathcal{O}_{X}(2 p+r)\right) \geq 2$. In any case, $3 p$ admits a $g_{3}^{1}$ for $X$. By Riemann-Roch, $2 p+q$ also yields a $g_{3}^{1}$. These must be two different $g_{3}^{1}$ 's, for $p \neq q$.

Since $X$ is not hyperelliptic, its canonical image is contained in a quadric surface $Q$ in $\mathbb{P}^{3}$. By Geometric Riemann-Roch, a section of a $g_{3}^{1}$ on $X$ corresponds to a line in $\mathbb{P}^{3}$ that intersects $Q$ at three points (counting with multiplicity). By Bézout, this line must be a ruling of $Q$. Since $X$ has two $g_{3}^{1}$ 's, $Q$ must be smooth and its two rulings correspond to the two $g_{3}^{1}$ 's. But the two lines spanned by the sections $2 p+q$ and $3 p$ cannot be both tangent to $X$ at the smooth point $p$, contradiction.

Proof of Theorem 1.2, Case $\Omega \mathcal{M}_{4}(5,1)$ We lift the Teichmüller curve $\bar{C}$ to $\overline{\mathcal{M}}_{4,1}$ using the 5 -fold zero of $\omega$. By the proposition $\bar{C} \cdot B N_{3,(2)}^{1}=0$, we have

$$
\bar{C} \cdot\left(4 \omega_{\text {rel }}+8 \lambda-\delta_{0}\right)=0 .
$$

By Proposition 4.8, we also have

$$
\bar{C} \cdot \omega_{\text {rel }}=\frac{\bar{C} \cdot \lambda-(\bar{C} \cdot \delta) / 12}{11 / 3} .
$$

Now the result follows by combining the two equalities.

\subsection{The stratum $\Omega \mathcal{M}_{4}(4,2)^{\text {even* }}$}

In the case $\Omega \mathcal{M}_{4}(4,2)^{\text {even }}$ we have

$$
L_{(4,2)^{\text {even }}}=32 / 15, \quad s_{(4,2)^{\text {even }}}=17 / 2, \quad c_{(4,2)^{\text {even }}}=68 / 45 .
$$

Based on numerical values on individual Teichmüller curves, we believe that the sum of Lyapunov exponents is nonvarying in this stratum. But we have not found a moduli space and a divisor to perform the desired disjointness argument.

\subsection{The stratum $\Omega \mathcal{M}_{4}(4,2)^{\text {odd } *}$}

In the case $\Omega \mathcal{M}_{4}(4,2)^{\text {odd }}$ we have

$$
\left.L_{(4,2) \text { odd }}=29 / 15, \quad s_{(4,2)}\right)^{\text {odd }}=236 / 29, \quad c_{(4,2)^{\text {odd }}}=59 / 45 .
$$


We also believe that the sum of Lyapunov exponents is nonvarying in this case. But we have not discovered a divisor that would do the job.

\subsection{The stratum $\Omega \mathcal{M}_{4}(3,3)^{\text {non-hyp }}$}

In the case $\Omega \mathcal{M}_{4}(3,3)^{\text {non-hyp }}$ we have

$$
L_{(3,3)^{\text {non-hyp }}}=2, \quad s_{(3,3)^{\text {non-hyp }}}=33 / 4, \quad c_{(3,3)^{\text {non-hyp }}}=11 / 8 .
$$

Proposition 6.4 Let $C$ be a Teichmüller curve generated by a flat surface $(X, \omega) \in$ $\Omega \mathcal{M}_{4}(3,3)^{\text {non-hyp }}$, lifted to $\overline{\mathcal{M}}_{4,2}$ (after a degree two base change). Then $\bar{C}$ does not intersect the divisor $\operatorname{Lin}_{3}^{1}$.

Proof Recall that $\operatorname{Lin}_{3}^{1} \subset \overline{\mathcal{M}}_{4,2}$ parameterizes pointed curves $(X, p, q)$ that admit a $g_{3}^{1}$ with a section vanishing at $p, q, r$ for some $r \in X$. Suppose $(X, p, q)$ is contained in the intersection of $\bar{C}$ with $\operatorname{Lin}_{3}^{1}$. Since $\omega_{X} \sim \mathcal{O}_{X}(3 p+3 q)$ and $h^{0}\left(\mathcal{O}_{X}(p+q+r)\right) \geq 2$, by Riemann-Roch we know that $h^{0}\left(\mathcal{O}_{X}(2 p+2 q-r)\right) \geq 2$. If $r \neq p, q$, then $h^{0}\left(\mathcal{O}_{X}(2 p+2 q)\right) \geq 3$, hence $2 p+q$ and $2 q+p$ both admit $g_{3}^{1}$. If $X$ is not hyperelliptic, using the canonical image of $X$ contained in a quadric in $\mathbb{P}^{3}$ and the preceding argument of rulings, one concludes that $2 p+q$ and $2 q+p$ span the same line (connecting $p, q$ ) on the quadric, contradiction. If $r=p$ or $q$, again, $2 p+q$ and $2 q+p$ both admit $g_{3}^{1}$ and consequently $X$ is hyperelliptic. But this stratum is nonhyperelliptic, and Proposition 3.4 yields the desired contradiction.

Proof of Theorem 1.2, Case $\Omega \mathcal{M}_{4}(3,3)$ Since we have $\bar{C} \cdot \operatorname{Lin}_{3}^{1}=0$, together with Proposition 4.8 and $\kappa_{(3,3)}=5 / 8$, the result follows immediately.

\subsection{The stratum $\Omega \mathcal{M}_{4}(2,2,2)^{\text {odd }}$}

In the case $\Omega \mathcal{M}_{4}(2,2,2)^{\text {odd }}$ we have

$$
L_{(2,2,2)^{\text {odd }}}=2, \quad s_{(2,2,2)^{\text {odd }}}=8, \quad c_{(2,2,2)} \text { odd }=4 / 3 .
$$

Note that by [33, Proposition 7] this stratum contains the hyperelliptic curves where all the zeros are fixed, but not those, where a pair of zeros are exchanged.

Proof of Theorem 1.2, Case $\Omega \mathcal{M}_{4}(2,2,2)^{\text {odd }} \quad$ We just need to apply Proposition 5.2 to obtain the result. It does apply to this case, because an odd theta characteristic on a genus four curve cannot have three or more sections by Clifford's theorem (Theorem 2.5). Note that such a theta characteristic $D$ is balanced, since $\operatorname{deg}\left(\left.D\right|_{Z}\right)=$ $w_{Z} / 2=w_{Z} \operatorname{deg}(D) /(2 g-2)$ for every component $Z$ of a stable curve $X_{\infty}$ over the Teichmüller curve. 


\subsection{The stratum $\Omega \mathcal{M}_{4}(3,2,1)$}

In the case $\Omega \mathcal{M}_{4}(3,2,1)$, we have

$$
L_{(3,2,1)}=25 / 12, \quad s_{(3,2,1)}=41 / 5, \quad c_{(3,2,1)}=205 / 144 .
$$

Proposition 6.5 Let $C$ be a Teichmüller curve generated by a flat surface $(X, \omega) \in$ $\Omega \mathcal{M}_{4}(3,2,1)$, lifted to $\overline{\mathcal{M}}_{4,3}$. Then $\bar{C}$ does not intersect the divisor $B N_{4,(1,1,2)}^{1}$.

Proof Recall the Brill-Noether divisor $B N_{4,(1,1,2)}^{1} \subset \overline{\mathcal{M}}_{4,3}$ parameterizes curves with a $g_{4}^{1}$ given by $p+q+2 r$. Suppose $(X, \omega)$ is contained in the intersection of $\bar{C}$ with $B N_{4,(1,1,2)}^{1}$, where $\operatorname{div}(\omega)=3 p+2 q+r$ and $p, q, r$ are the (ordered) marked points. By the fact that $h^{0}\left(\mathcal{O}_{X}(p+q+2 r)\right) \geq 2$ and Riemann-Roch, we have $h^{0}\left(\mathcal{O}_{X}(2 p+q-r)\right) \geq 1$. Consequently $h^{0}\left(\mathcal{O}_{X}(2 p+q)\right) \geq 2$ and by Riemann-Roch again, we have $h^{0}\left(\mathcal{O}_{X}(p+q+r)\right) \geq 2$. Note that if $2 p+q \sim p+q+r$, then $p \sim r$, which is impossible. If these are two different $g_{3}^{1}$ 's and $X$ is smooth, or stable but nonhyperelliptic and at least three-connected, then its canonical map is an embedding. Consequently both $p$ and $q$ lie on two different rulings of the quadric containing the canonical image of $X$. This is impossible.

Since hyperelliptic stable fibers cannot occur by Proposition 3.4, the last case to be excluded consists of a stable curve which is only two-connected. Given the constraints in Corollary 3.2, there are two possible types for such a stable curve. First, there are two irreducible nodal curves $X_{1}$ and $X_{2}$ of arithmetic genus one and two, respectively, joined at two nodes $\{x, y\}$ with $q$ lying on $X_{1}$ and $p$ and $r$ lying on $X_{2}$. Second, there are irreducible nodal curves $X_{1}, X_{0}$ and $Y_{1}$, the index specifying the arithmetic genus with $p$ on $Y_{1}, q$ on $X_{1}$ and $r$ on $X_{0}$, whose intersection is given by $Y_{1} \cdot X_{1}=\{x\}$, $Y_{1} \cdot X_{0}=\left\{z_{1}, z_{2}\right\}, X_{1} \cdot X_{0}=\{y\}$.

For the first type, consider the linear system $\left|\mathcal{O}_{X}(2 p+q)\right|$. Since $p$ and $q$ lie on different components of the stable curve, $q$ has to be a base point of this linear system. Hence $\omega_{X_{2}}(x+y) \sim \mathcal{O}_{X_{2}}(4 p)$, ie $p$ is a Weierstrass point for the line bundle $\omega_{X_{2}}(x+y)$. By the same argument $q$ is a base point of $\left|\mathcal{O}_{X}(p+q+r)\right|$, hence $\omega_{X_{2}}(x+y) \sim \mathcal{O}_{X_{2}}(2 p+2 r)$. Since $p \neq r$, this implies that $p$ is not a Weierstrass point and we obtain the desired contradiction.

For the second type, the condition $h^{0}\left(\mathcal{O}_{X}(p+q+r)\right) \geq 2$ provides an immediate contradiction, since all three points lie on different components of the stable curve.

Proof of Theorem 1.2, Case $\Omega \mathcal{M}_{4}(3,2,1)$ Recall the divisor class of $B N_{(1,1,2)}^{1}$ in (6). By the fact that $\bar{C} \cdot B N_{4,(1,1,2)}^{1}=0$ together with Proposition 4.8, the result follows directly. 


\subsection{Varying sum in the stratum $\Omega \mathcal{M}_{4}(2,2,2)^{\text {even }}$}

In the case $\Omega \mathcal{M}_{4}(2,2,2)^{\text {even }}$, we have

$$
L_{(2,2,2)^{\mathrm{even}}}=166 / 75, \quad s_{(2,2,2)^{\mathrm{even}}}=696 / 83, \quad c_{(2,2,2)^{\mathrm{even}}}=116 / 75 .
$$

Note that by [33, Proposition 7] this stratum contains the hyperelliptic curves where a pair of zeros are exchanged, but not those, where all the zeros are fixed. For Teichmüller curves $C^{\text {hyp }}$ contained in the locus of hyperelliptic flat surfaces within this stratum, we have

$$
L\left(C^{\text {hyp }}\right)=7 / 3 .
$$

Examples The square-tiled surface $\left(X: y^{6}=x(x-1)(x-t), \omega=d x / y\right)$ found by Forni and Matheus [23] has maximally degenerate Lyapunov spectrum, ie $L(C)=1$.

Proposition 6.6 A Teichmüller curve $C$ generated by a nonhyperelliptic flat surface $(X, \omega) \in \Omega \overline{\mathcal{M}}_{4}(2,2,2)^{\text {even }}$ has

$$
L(C) \leq 16 / 7
$$

In particular the sum of Lyapunov exponents of any Teichmüller curve generated by a nonhyperelliptic flat surface in this stratum is strictly smaller than the sum of Lyapunov exponents of any Teichmüller curve generated by a hyperelliptic flat surface in this stratum.

Proof Recall the divisor class in (11) of the Gieseker-Petri divisor $G P$ on $\overline{\mathcal{M}}_{4}$. It has slope equal to $17 / 2$. Hence if $C$ is not entirely contained in this divisor, we have $s(C) \leq 17 / 2$ by Lemma 4.7, which translates into $L(C) \leq 16 / 7$.

If $C$ is contained in $G P$, we try to intersect $C$ with the Brill-Noether divisor $B N_{4,(3,1)}^{1}$. If $C$ is not contained in $B N_{4,(3,1)}^{1}$, using Proposition 4.8 with $\kappa=2 / 3$ we obtain a better bound $L(C) \leq 2$.

Suppose that a generic surface $(X, \omega)$ parameterized by $C$ lies in $B N_{4,(3,1)}^{1}$. By definition, we have $h^{0}\left(\mathcal{O}_{X}(3 p+q)\right) \geq 2$, which implies $h^{0}\left(\mathcal{O}_{X}(q+2 r-p)\right) \geq 1$ by Riemann-Roch. Consequently we have $h^{0}\left(\mathcal{O}_{X}(2 r+q)\right) \geq 2$ and $h^{0}\left(\mathcal{O}_{X}(2 p+q)\right) \geq 2$. Since we excluded hyperelliptic $X$, the assumption that $X$ is parameterized in $G P$ implies that $2 r+q \sim 2 p+q$. Hence $2 p \sim 2 r$, which contradicts the assumption that $X$ is not hyperelliptic. 


\subsection{Varying sum in the stratum $\Omega \mathcal{M}_{4}(4,1,1)$}

In this stratum we have

$$
L_{(4,1,1)}=1137 / 550 \approx 2.06727, \quad s_{(4,1,1)}=3118 / 379, \quad c_{(4,1,1)}=1559 / 1100 .
$$

The stratum contains the locus of hyperelliptic flat surfaces coming from $\mathcal{Q}\left(3,2,-1^{9}\right)$. Hence for a Teichmüller curve $C^{\text {hyp }}$ in this locus, the sum of Lyapunov exponents is

$$
L\left(C^{\text {hyp }}\right)=23 / 10 \text {. }
$$

Examples A Teichmüller curve $C$ generated by the square-tiled surface with

$$
\left(\pi_{r}=(12)(3)(4)(5)(67)(8)(910), \pi_{u}=(132456879)(10)\right)
$$

has $L(C)=1043 / 530 \approx 1.96792$.

A Teichmüller curve $C$ generated by the square-tiled surface with

$$
\left(\pi_{r}=(12)(3)(4)(5)(6)(7,8)(9)(1011), \pi_{u}=(13)(2,4,5,6,7,9)(810)(11)\right)
$$

has $L(C)=267163 / 129510 \approx 2.06287$.

This stratum also contains Teichmüller curves $C$ generated by square-tiled surfaces with

$$
L(C) \in\{1043 / 530 \approx 1.96792,579 / 290,4101 / 1990,1799 / 870 \approx 2.06782,23 / 10\} .
$$

Proposition 6.7 A Teichmüller curve $C$ generated by a nonhyperelliptic flat surface $(X, \omega) \in \Omega \mathcal{M}_{4}(4,1,1)$ has

$$
L(C) \leq 21 / 10 \text {. }
$$

In particular the sum of Lyapunov exponents of any Teichmüller curve generated by a nonhyperelliptic flat surface in this stratum is strictly smaller than the sum of Lyapunov exponents of any Teichmüller curve generated by a hyperelliptic flat surface in this stratum.

Proof By the same argument as in the proof for the stratum $\Omega \mathcal{M}_{3}(2,1,1)$ we may pass to an unramified covering and label the zeros of $\omega$ such that $\omega_{X} \sim \mathcal{O}_{X}(4 p+q+r)$ along the whole family. Using $p$ and $q$ we lift this covering to a curve in $\overline{\mathcal{M}}_{4,2}$ that we continue to call $C$. We will show that $C$ is not entirely contained in the divisor $B N_{4,(2,2)}^{1}$, provided that $X$ is not hyperelliptic. Then $\bar{C} \cdot B N_{4,(2,2)}^{1} \geq 0$ together with Proposition 4.8 using $\kappa_{(4,1,1)}=39 / 60$ implies the claim.

Suppose a generic flat surface $(X, \omega)$ in $C$ is contained in $B N_{4,(2,2)}^{1}$. Then by definition $h^{0}\left(\mathcal{O}_{X}(2 p+2 q)\right) \geq 2$ and by Riemann-Roch $h^{0}\left(\mathcal{O}_{X}(2 p-q+r)\right) \geq 1$. Hence 
$h^{0}\left(\mathcal{O}_{X}(2 p+r)\right) \geq 2$ and by Riemann-Roch again $h^{0}\left(\mathcal{O}_{X}(2 p+q)\right) \geq 2$. Since $X$ is not hyperelliptic, we consider the quadric surface containing its canonical image. The ruling that is tangent to $X$ at $p$ intersects $X$ at a third point. This point has to be both $q$ and $r$ due to the $g_{3}^{1}$ 's given by $2 p+q$ and $2 p+r$, which is absurd for $q \neq r$.

\subsection{Varying sum in the stratum $\Omega \mathcal{M}_{4}(3,1,1,1)$}

In this stratum we have

$$
L_{(3,1,1,1)}=66 / 31 \approx 2.12903, \quad s_{(3,1,1,1)}=65 / 8, \quad c_{(3,1,1,1)}=715 / 496 .
$$

This stratum does not contain any submanifolds obtained by double covering constructions.

Examples The Teichmüller curve $C$ generated by the square-tiled surface with

$$
\left(\pi_{r}=(12345678910), \pi_{u}=(14583610)(279)\right)
$$

has $L(C)=514 / 243 \approx 2.11523$.

The Teichmüller curve $C$ generated by the square-tiled surface with

$$
\left(\pi_{r}=(1234567891011), \pi_{u}=(1458102711)(369)\right)
$$

has $L(C)=1531 / 720 \approx 2.12639$.

There exist Teichmüller curves $C$ generated by square-tiled surfaces in this stratum with

$$
L(C) \in\{241 / 114 \approx 2.114035,72167 / 33984,1531 / 720 \approx 2.1263\} .
$$

Proposition 6.8 A Teichmüller curve $C$ which is generated by a flat surface $(X, \omega) \in$ $\Omega \overline{\mathcal{M}}_{4}(3,1,1,1)$ has

$$
L(C) \leq 7 / 3
$$

Proof The proof is identical to the one given below for the stratum $\Omega \mathcal{M}_{4}(2,1,1,1,1)$ using two different lifts to $\overline{\mathcal{M}}_{4,3}$ and the divisor $B N_{4,(1,1,2)}^{1}$ (see Section 6.13). 


\subsection{Varying sum in the stratum $\Omega \mathcal{M}_{4}(2,2,1,1)$}

In this stratum we have

$L_{(2,2,1,1)}=5045 / 2358 \approx 2.13952, s_{(2,2,1,1)}=8178 / 1009, \quad c_{(2,2,1,1)}=6815 / 4716$.

The stratum contains two loci of hyperelliptic flat surfaces. One of them corresponds to the orientation double covers of $\mathcal{Q}\left(4,2,-1^{10}\right)$, hence for a Teichmüller curve $C$ in this locus, the sum of Lyapunov exponents is $L(C)=5 / 2$. In this locus, the zeros are permuted in pairs by the hyperelliptic involution.

The second one corresponds to $\mathcal{Q}\left(2,1,1,-1^{8}\right)$, hence for a Teichmüller curve $C$ in this locus, the sum of Lyapunov exponents is $L(C)=13 / 6 \approx 2.16$.

Examples The Teichmüller curve $C$ generated by the square-tiled surface with

$$
\left(\pi_{r}=(12)(3)(4)(5)(67)(8)(9)(1011)(12), \pi_{u}=(134)(256789101112)\right)
$$

has $L(C)=3313 / 1590 \approx 2.083$.

The Teichmüller curve $C$ generated by the square-tiled surface with

$$
\left(\pi_{r}=(12)(3)(4)(56)(7)(89)(1011), \pi_{u}=(134578)(26910)(11)\right)
$$

has $L(C)=4919 / 2312 \approx 2.1275$.

There exist Teichmüller curves $C$ generated by square-tiled surfaces in this stratum with

$$
L(C) \in\{3313 / 1590 \approx 2.083,157 / 75,273529 / 128580,4919 / 2312 \approx 2.1275\} .
$$

Proposition 6.9 A Teichmüller curve $C$ generated by a nonhyperelliptic flat surface $(X, \omega) \in \Omega \overline{\mathcal{M}}_{4}(2,2,1,1)$ has

$$
L(C) \leq 13 / 6
$$

In particular the sum of Lyapunov exponents of any Teichmüller curve generated by a nonhyperelliptic flat surface in this stratum is strictly smaller than the sum of Lyapunov exponents of any Teichmüller curve generated by a hyperelliptic flat surface where the four zeros are permuted in pairs.

Proof By the same argument as in the proof for the stratum $\Omega \mathcal{M}_{3}(2,1,1)$ we may pass to an unramified covering and label the zeros of $\omega$ such that $\omega_{X} \sim \mathcal{O}_{X}(2 p+2 q+r+s)$ along the whole family. We lift this covering to a curve in $\overline{\mathcal{M}}_{4,3}$ by marking $p, q, r$ and continue to call it $C$. We will show that $C$ is not entirely contained in the 
divisor $B N_{4,(1,1,2)}^{1}$. Then $\bar{C} \cdot B N_{4,(1,1,2)}^{1} \geq 0$ together with Proposition 4.8 implies this proposition.

Suppose a generic flat surface $(X, \omega)$ parameterized by $C$ is contained in $B N_{4,(1,1,2)}^{1}$. Then by definition we have $h^{0}\left(\mathcal{O}_{X}(p+q+2 r)\right) \geq 2$ and by Riemann-Roch we have $h^{0}\left(\mathcal{O}_{X}(p+q-r+s)\right) \geq 1$. Hence $h^{0}\left(\mathcal{O}_{X}(p+q+s)\right) \geq 2$ and by Riemann-Roch again $h^{0}\left(\mathcal{O}_{X}(p+q+r)\right) \geq 2$. For $X$ nonhyperelliptic this means that on the quadric containing the canonical image of $X$ in $\mathbb{P}^{3}$, there are two rulings passing through $p$ and $q$ (hence they are the same ruling), one intersecting the curve moreover at $r$ and the other intersecting the curve moreover at $s$. This is impossible for $r \neq s$.

\subsection{Varying sum in the stratum $\Omega \mathcal{M}_{4}(2,1,1,1,1)$}

In this stratum we have

$L_{(2,1,1,1,1)}=131 / 60 \approx 2.18333, \quad s_{(2,1,1,1,1)}=1052 / 131, \quad c_{(2,1,1,1,1)}=263 / 180$.

It contains the locus of hyperelliptic flat surfaces corresponding to $\mathcal{Q}\left(3,2,2,-1^{11}\right)$. Hence for a Teichmüller curve $C$ in this locus, the sum of Lyapunov exponents is $L(C)=14 / 5=2.8$.

Examples The Teichmüller curve $C$ generated by the square-tiled surface with

$$
\left(\pi_{r}=(12)(3)(4)(5,6)(7)(8)(910)(1112)(13), \pi_{u}=(13245768911101213)\right),
$$

has $L(C)=268 / 129 \approx 2.0775$.

The Teichmüller curve $C$ generated by the square-tiled surface with

$$
\left(\pi_{r}=(12)(3)(4)(5)(6)(78)(910)(11)(1213), \pi_{u}=(13)(2456791181210)(13)\right),
$$

has $L(C)=207826 / 95511 \approx 2.1759$.

There exist Teichmüller curves $C$ generated by square-tiled surfaces in this stratum with

$$
L(C) \in\{268 / 129 \approx 2.0775,239 / 114,4031 / 1923,207826 / 95511 \approx 2.175\} .
$$

Proposition 6.10 A Teichmüller curve $C$ generated by a nonhyperelliptic flat surface $(X, \omega) \in \Omega \overline{\mathcal{M}}_{4}(2,1,1,1,1)$ has

$$
L(C) \leq 7 / 3 .
$$

In particular the sum of Lyapunov exponents of any Teichmüller curve generated by a nonhyperelliptic flat surface in this stratum is strictly smaller than the sum of Lyapunov exponents of any Teichmüller curve generated by a hyperelliptic flat surface in this stratum. 
Proof By the same argument as in the proof for the stratum $\Omega \mathcal{M}_{3}(2,1,1)$, we may pass to an unramified covering and label the zeros of $\omega$ so $\omega_{X} \sim \mathcal{O}_{X}(2 p+q+r+s+u)$ along the whole family. First, using $p, q$ and $r$ we lift this covering to a curve in $\overline{\mathcal{M}}_{4,3}$ that we continue to call $C$.

If $C$ is not entirely contained in the divisor $B N_{4,(1,2,1)}^{1}$, then $\bar{C} \cdot B N_{4,(1,2,1)}^{1} \geq 0$ together with Proposition 4.8 using $\kappa_{(2,1,1,1,1)}=13 / 18$ implies the claim. If $C$ is entirely contained in the divisor $B N_{4,(1,2,1)}^{1}$, we can lift $C$ to $\overline{\mathcal{M}}_{4,3}$ alternatively by marking $p, q$ and $r$. Again, if $C$ is not contained in $B N_{4,(1,2,1)}^{1}$, the claim holds.

Suppose that $C$ is contained in the Brill-Noether divisor for both lifts. Then for $(X, \omega)$ parameterized in $C$, by definition we have $h^{0}\left(\mathcal{O}_{X}(p+2 q+r)\right) \geq 2$, consequently we obtain $h^{0}\left(\mathcal{O}_{X}(s+u+p-q)\right) \geq 1$ and $h^{0}\left(\mathcal{O}_{X}(s+u+p)\right) \geq 2$. For the second lift we deduce from $h^{0}\left(\mathcal{O}_{X}(p+2 q+u)\right) \geq 2$ that $h^{0}\left(\mathcal{O}_{X}(s+r+p)\right) \geq 2$. Since $X$ is not hyperelliptic, the canonical map is an embedding and its image lies on a quadric surface in $\mathbb{P}^{3}$. Then the unique line on the quadric passing through $s$ and $p$ cannot have a third intersection point with $C$ at both $r$ and $u$ for $r \neq u$.

\subsection{Varying sum in the stratum $\Omega \mathcal{M}_{4}(1,1,1,1,1,1)$}

In this stratum we have

$$
L_{(1,1,1,1,1,1)}=\frac{839}{377} \approx 2.22546, \quad s_{(1,1,1,1,1,1)}=\frac{6675}{839}, \quad c_{(1,1,1,1,1,1)}=\frac{2225}{1508} .
$$

The stratum contains the locus of hyperelliptic flat surfaces corresponding to the stratum $\mathcal{Q}\left(2,2,2,-1^{10}\right)$. Hence for a Teichmüller curve $C$ in this locus we have $L(C)=5 / 2$.

Examples The Teichmüller curve $C$ generated by the square-tiled surface with $\left(\pi_{r}=(12)(3)(4)(5)(6)(78)(9)(10)(1112)(13)(14), \pi_{u}=(132456798101113)(1214)\right)$ has $L(C)=125 / 58 \approx 2.15517$.

The Teichmüller curve $C$ generated by the square-tiled surface with

$$
\left(\pi_{r}=(12)(3)(4)(56)(7)(8)(910)(11)(12), \pi_{u}=(132457689111012)\right),
$$

has $L(C)=9 / 4=2.25$.

There exist Teichmüller curves $C$ generated by square-tiled surfaces in this stratum with

$$
L(C) \in\{125 / 58 \approx 2.15517,419 / 194,1019 / 470,8498 / 3867 \approx 2.1975,9 / 4\} .
$$


Proposition 6.11 A Teichmüller curve $C$ which is generated by a flat surface $(X, \omega) \in$ $\Omega \overline{\mathcal{M}}_{4}(1,1,1,1,1,1)$ has

$$
L(C) \leq 5 / 2
$$

Proof The argument is completely analogous to the stratum $\Omega \overline{\mathcal{M}}_{4}(2,1,1,1,1)$.

\section{Genus five}

In genus 5 only few strata have a nonvarying sum of Lyapunov exponents. We summarize the results in Figures 4 and 5. Contrary to genus 4 we do not give an upper bound for the sum in all the (components of) strata where the sum is varying but provide only one example which often comes from the locus of hyperelliptic flat surfaces.

\subsection{The stratum $\Omega \mathcal{M}_{5}(8)^{\text {even }}$}

In the case $\Omega \mathcal{M}_{5}(8)^{\text {even }}$ we have

$$
L_{(8)^{\text {even }}}=20 / 9, \quad s_{(8)^{\text {even }}}=8, \quad c_{(8)^{\text {even }}}=50 / 27 .
$$

Proposition 7.1 Let $C$ be a Teichmüller curve generated by a flat surface which is in $\Omega \mathcal{M}_{5}(8)^{\text {even }}$. Then $\bar{C}$ does not intersect the Brill-Noether divisor $B N_{3}^{1}$ on $\overline{\mathcal{M}}_{5}$.

Proof Teichmüller curves in this stratum are disjoint from the hyperelliptic locus even at the boundary of $\overline{\mathcal{M}}_{5}$, since the hyperelliptic component is a different component and by Proposition 3.4. Suppose $(X, \omega)$ is a flat surface contained in the intersection of $\bar{C}$ and $B N_{3}^{1}$. Since $X$ is trigonal (possibly nodal but irreducible) and is not hyperelliptic, its canonical image lies on a cubic scroll surface in $\mathbb{P}^{4}$ whose rulings are spanned by the sections of the $g_{3}^{1}$ (see eg [40, Section 2.10]). This scroll surface can be either smooth or singular, corresponding to Hirzebruch surfaces $F_{n}$ of two types, respectively (see Beauville [4, Chapter IV] or Coskun [11, Section 2] for preliminaries on Hirzebruch surfaces). Here we follow the notation in [11].

Suppose the scroll surface is smooth as the embedding of the Hirzebruch surface $F_{1}$ by the linear system $|e+2 f|$, where

$$
e^{2}=-1, \quad e \cdot f=1, \quad f^{2}=0 .
$$

Then $X$ has class $3 e+5 f$. Note that $4 p$ admits a $g_{4}^{1}$, which comes from the projection of $X$ from a plane $\Lambda$ to a line in $\mathbb{P}^{4}$. This plane $\Lambda$ intersects $X$ at greater than or equal to 4 points (with multiplicity) and the intersection contains the residual $4 p$. But $F_{1}$ 


\begin{tabular}{|c|c|c|c|c|c|c|}
\hline \multirow{3}{*}{$\begin{array}{c}\text { Degrees } \\
\text { of } \\
\text { zeros } \\
\left(d_{1}, \ldots, d_{n}\right)\end{array}$} & \multirow{3}{*}{$\begin{array}{l}\text { Hyperelliptic } \\
\text { or spin } \\
\text { structure }\end{array}$} & \multicolumn{5}{|c|}{ Lyapunov exponents } \\
\hline & & \multicolumn{2}{|c|}{ Component } & \multicolumn{3}{|c|}{ Teichmüller curves } \\
\hline & & $\approx$ & $\sum_{j=1}^{g} \lambda_{j}$ & $\approx$ & $\sum_{j=1}^{g} \lambda_{j}$ & Reference \\
\hline (8) & hyperelliptic & 2.777778 & $\frac{25}{9}$ & \multicolumn{2}{|c|}{ Nonvarying } & Theorem 4.2 \\
\hline (8) & even & 2.222222 & $\frac{20}{9}$ & \multicolumn{2}{|c|}{ Nonvarying } & Section 7.1 \\
\hline (8) & odd & 2.111111 & $\frac{19}{9}$ & \multicolumn{2}{|c|}{ Nonvarying } & Section 7.2 \\
\hline$(7,1)$ & - & 2.227022 & $\frac{2423}{1088}$ & 2.229062 & $\frac{7133}{3200}$ & (18) \\
\hline$(6,2)$ & even & 2.301983 & $\frac{178429}{77511}$ & 2.619047 & $\frac{55}{21}$ & $\mathcal{Q}\left(5,1,-1^{10}\right)$ \\
\hline$(6,2)$ & odd & 2.190476 & $\frac{46}{21}$ & \multicolumn{2}{|c|}{ Nonvarying? } & - \\
\hline$(6,1,1)$ & - & 2.285384 & $\frac{59332837}{25961866}$ & 2.785714 & $\frac{39}{14}$ & $\mathcal{Q}\left(5,2,-1^{11}\right)$ \\
\hline$(5,3)$ & - & 2.250000 & $\frac{9}{4}$ & \multicolumn{2}{|c|}{ Nonvarying } & Section 7.3 \\
\hline$(5,2,1)$ & - & 2.300563 & $\frac{4493}{1953}$ & 2.302594 & $\frac{48541}{21081}$ & (19) \\
\hline$(5,1,1,1)$ & - & 2.340909 & $\frac{103}{44}$ & 2.337802 & $\frac{12381}{5296}$ & (20) \\
\hline$(4,4)$ & hyperelliptic & 3.000000 & 3 & \multicolumn{2}{|c|}{ Nonvarying } & Theorem 4.2 \\
\hline$(4,4)$ & even & 2.311111 & $\frac{104}{45}$ & 2.400000 & $\frac{12}{5}$ & (21) \\
\hline$(4,4)$ & odd & 2.191613 & $\frac{228605}{104309}$ & 2.600000 & $\frac{13}{5}$ & $\mathcal{Q}\left(3,3,-1^{10}\right)$ \\
\hline$(4,3,1)$ & - & 2.306255 & $\frac{438419}{190100}$ & 2.302715 & $\frac{777627}{337700}$ & (23) \\
\hline$(4,2,2)$ & even & 2.374007 & $\frac{34981}{14735}$ & 2.800000 & $\frac{14}{5}$ & $\mathcal{Q}\left(4,3,-1^{11}\right)$ \\
\hline$(4,2,2)$ & odd & 2.260315 & $\frac{538102}{238065}$ & 2.466666 & $\frac{37}{15}$ & $\mathcal{Q}\left(3,1,1,-1^{9}\right)$ \\
\hline$(4,2,1,1)$ & - & 2.354799 & $\frac{646039}{274350}$ & 2.633333 & $\frac{79}{30}$ & $\mathcal{Q}\left(3,2,1,-1^{10}\right)$ \\
\hline
\end{tabular}

Figure 4: Varying and nonvarying sums in genus five, part I 


\begin{tabular}{|c|c|c|c|c|c|c|}
\hline \multirow{3}{*}{$\begin{array}{c}\text { Degrees } \\
\text { of } \\
\text { zeros } \\
\left(d_{1}, \ldots, d_{n}\right)\end{array}$} & \multirow{3}{*}{$\begin{array}{c}\text { Hyperelliptic } \\
\text { or spin } \\
\text { structure }\end{array}$} & \multicolumn{5}{|c|}{ Lyapunov exponents } \\
\hline & & \multicolumn{2}{|c|}{ Component } & \multicolumn{3}{|c|}{ Teichmüller curves } \\
\hline & & $\approx$ & $\sum_{j=1}^{g} \lambda_{j}$ & $\approx$ & $\sum_{j=1}^{g} \lambda_{j}$ & Reference \\
\hline$\left(4,1^{4}\right)$ & - & 2.393586 & $\frac{640763}{267700}$ & 2.800000 & $\frac{14}{5}$ & $\mathcal{Q}\left(3,2,2,-1^{11}\right)$ \\
\hline$(3,3,2)$ & - & 2.318020 & $\frac{61307}{26448}$ & 2.833333 & $\frac{17}{6}$ & $\mathcal{Q}\left(6,1,-1^{11}\right)$ \\
\hline$(3,3,1,1)$ & - & 2.358542 & $\frac{47435}{20112}$ & 3.000000 & 3 & $\mathcal{Q}\left(6,2,-1^{12}\right)$ \\
\hline$(3,2,2,1)$ & - & 2.366588 & $\frac{6049}{2556}$ & 2.362268 & $\frac{2041}{864}$ & (24) \\
\hline$\left(3,2,1^{3}\right)$ & - & 2.405498 & $\frac{700}{291}$ & 2.398764 & $\frac{3495}{1457}$ & (25) \\
\hline$\left(3,1^{5}\right)$ & - & 2.443023 & $\frac{2101}{860}$ & 2.431085 & $\frac{77785}{31996}$ & (26) \\
\hline$(2,2,2,2)$ & even & 2.434379 & $\frac{2096}{861}$ & 2.666666 & $\frac{8}{3}$ & $\mathcal{Q}\left(4,1,1,-1^{10}\right)$ \\
\hline$(2,2,2,2)$ & odd & 2.319961 & $\frac{355309}{153153}$ & 2.333333 & $\frac{7}{3}$ & $\mathcal{Q}\left(1,1,1,1,-1^{8}\right)$ \\
\hline$\left(2^{3}, 1^{2}\right)$ & - & 2.413574 & $\frac{79981}{33138}$ & 2.833333 & $\frac{17}{6}$ & $\mathcal{Q}\left(4,2,1,-1^{11}\right)$ \\
\hline$\left(2,2,1^{4}\right)$ & - & 2.451217 & $\frac{266761}{108828}$ & 2.666666 & $\frac{8}{3}$ & $\mathcal{Q}\left(2,2,1,1,-1^{10}\right)$ \\
\hline$\left(2,1^{6}\right)$ & - & 2.487756 & $\frac{35861}{14415}$ & 2.833333 & $\frac{17}{6}$ & $\mathcal{Q}\left(2,2,2,1,-1^{11}\right)$ \\
\hline$\left(1^{8}\right)$ & - & 2.523451 & $\frac{235761}{93428}$ & 3.000000 & 3 & $\mathcal{Q}\left(2,2,2,2,-1^{12}\right)$ \\
\hline
\end{tabular}

Figure 5: Varying and nonvarying sums in genus five, part II

has degree three, so the intersection $F_{1} \cap \Lambda$ consists of a curve $B$ with possibly finitely many points outside $B$. If $B$ is a ruling, then $B \cdot X=3$ and $\Lambda$ also intersects $X$ at a point outside $B$, such that $\Lambda$ is spanned by $B$ and that point. Then we cannot have $4 p \subset \Lambda \cap X$, contradiction. If $B$ has higher degree, it can only be a conic (or its degeneration) of class $e+f$. Then $B \cdot X=5$, so $\Lambda \cap X=4 p+q$ admits a $g_{5}^{2}$ by Geometric Riemann-Roch. For $q \neq p$ we obtain $h^{0}\left(\mathcal{O}_{X}(4 p-q)\right)=2$ and hence $h^{0}\left(\mathcal{O}_{X}(4 p)\right)=3$, contradiction. For $q=p$, the residual $3 p$ admits a $g_{3}^{1}$, so it gives rise to a ruling $L$ on the cubic scroll. Then $L$ and $B$ are both tangent to $X$ at $p$. But $L \cdot B=f \cdot(e+f)=1$, leading to a contradiction. 
If the scroll is singular, it is isomorphic to $F_{3}$ by the linear system $|e+3 f|$, where

$$
e^{2}=-3, \quad e \cdot f=1, \quad f^{2}=0 .
$$

Since $X \cdot f=3$ and $X \cdot(e+3 f)=8$, it has class $3 e+8 f$. Then $X \cdot e=-1$, which implies that $X$ consists of $e$ union a curve of class $2 e+8 f$, contradicting the irreducibility of $X$.

Proof of Theorem 1.3, Case $\Omega \mathcal{M}_{5}(8)^{\text {even }} \quad$ By the proposition we have $\bar{C} \cdot B N_{3}^{1}=0$. Since this divisor has slope equal to 8 by (4), the Teichmüller curve $C$ has the same slope $s(C)=8$.

\subsection{The stratum $\Omega \mathcal{M}_{5}(8)^{\text {odd }}$}

In the case $\Omega \mathcal{M}_{5}(8)^{\text {odd }}$ we have

$$
L_{(8)^{\text {odd }}}=19 / 9, \quad s_{(8)^{\text {odd }}}=148 / 19, \quad c_{(8)}{ }^{\text {odd }}=37 / 27 .
$$

Proposition 7.2 Let $C$ be a Teichmüller curve generated by a flat surface $(X, \omega) \in$ $\Omega \mathcal{M}_{5}(8)^{\text {odd }}$ lifted to $\overline{\mathcal{M}}_{5,1}$ using the zero of $\omega$. Then $\bar{C}$ does not intersect the divisor $\operatorname{Nfold}_{5,4}^{1}(1)$.

Proof Suppose that $(X, \omega)$ is contained in the intersection of $\bar{C}$ with $\operatorname{Nfold}_{5,4}^{1}(1)$. Note that $X$ is not hyperelliptic, as this component and the hyperelliptic component are disjoint and by Proposition 3.4. Recall that $\operatorname{Nfold}_{5,4}^{1}(1) \subset \overline{\mathcal{M}}_{5,1}$ parameterizes curves that admit a $g_{4}^{1}$ given by $3 p+q$, where $p$ is the marked point and $q$ is a random point. Then it implies that $h^{0}\left(\mathcal{O}_{X}(3 p+q)\right)=2$ and $h^{0}\left(\mathcal{O}_{X}(4 p)\right)=1$ by Clifford's theorem, hence we have $q \neq p$. By Riemann-Roch, we have $h^{0}\left(\mathcal{O}_{X}(5 p-q)\right)=2$. Since $h^{0}\left(\mathcal{O}_{X}(5 p)\right)=2=h^{0}\left(\mathcal{O}_{X}(5 p-q)\right)$, it implies that $q$ is a base point of $\left|\mathcal{O}_{X}(5 p)\right|$, which is impossible.

Proof of Theorem 1.3, Case $\Omega \mathcal{M}_{5}(8)^{\text {odd }}$ By Proposition 7.2 we have

$$
\bar{C} \cdot \operatorname{Nfold}_{5,4}^{1}(1)=0 .
$$

It now suffices to plug the result of Proposition 4.8 with $m_{1}=8$ into the divisor class of Nfold $_{5,4}^{1}(1)$ in (9) to obtain the desired numbers. 


\subsection{The stratum $\Omega \mathcal{M}_{5}(5,3)$}

In the case $\Omega \mathcal{M}_{g}(5,3)$ we have

$$
L_{(5,3)}=9 / 4, \quad s_{(5,3)}=209 / 27, \quad c_{(5,3)}=209 / 144 .
$$

Proposition 7.3 Let $C$ be a Teichmüller curve generated by a flat surface $(X, \omega) \in$ $\Omega \mathcal{M}_{5}(5,3)$, lifted to $\overline{\mathcal{M}}_{5,2}$ by the zeros of $\omega$. Then $\bar{C}$ does not intersect the divisor $B N_{4,(1,2)}^{1}$.

Proof Note that the degenerate fibers of the family over $\bar{C}$ are either irreducible or consist of two components connected by an odd number $(\geq 3)$ of nodes by Corollary 3.3. Moreover by Proposition 3.4 the degenerate fibers are not hyperelliptic. Consequently the dual graph of $X$ is three-connected and $\omega_{X}$ is very ample by Proposition 2.6.

Suppose that contrary to the claim, $(X, p, q)$ is contained in the intersection of $\bar{C}$ and $B N_{4,(1,2)}^{1}$, ie $h^{0}\left(\mathcal{O}_{X}(2 p+q+r)\right)=2$ for some $r \in X$. Since $\omega_{X} \sim \mathcal{O}_{X}(5 p+3 q)$, by Riemann-Roch we have $h^{0}\left(\mathcal{O}_{X}(3 p+2 q-r)\right)=2$. For $r=p$ or $q$, these equalities reduce to $h^{0}\left(\mathcal{O}_{X}(2 p+2 q)\right)=2$ and $h^{0}\left(\mathcal{O}_{X}(3 p+q)\right)=2$. If $r \neq p, q$, then $r$ is not a base point of $\left|\mathcal{O}_{X}(3 p+2 q)\right|$, hence $h^{0}\left(\mathcal{O}_{X}(3 p+2 q)\right)=3$ and $h^{0}\left(\mathcal{O}_{X}(2 p+q)\right)=2$. Then we still have $h^{0}\left(\mathcal{O}_{X}(2 p+2 q)\right)=h^{0}(3 p+q) \geq 2$, hence they are equal to 2 by Clifford's theorem. In any case, $3 p+q$ and $2 p+2 q$ span two different planes with the corresponding contact orders at $p$ and $q$ to the canonical image of $X$ in $\mathbb{P}^{4}$. The two planes contain a common line spanned by $p, q$ whose intersection with $X$ is $2 p+q$. By Geometric Riemann-Roch, $2 p+q$ gives rise to a $g_{3}^{1}$, hence $X$ is trigonal.

For a trigonal genus 5 curve $X$ with $\omega_{X}$ very ample, as we have seen in Section 7.1, its canonical image is contained in a cubic scroll surface in $\mathbb{P}^{4}$. The residual $g_{5}^{2}$ given by $3 p+2 q$ maps $X$ to a plane quintic $Y$, whose image differs from $X$ at a double point $u$ (like a node or cusp), for the arithmetic genus of $Y$ is 6 . The unique $g_{3}^{1}$ on $X$ is given by intersections of lines passing through $u$ with $Y$ (subtracting $2 u$ from the base locus). But $2 p+q$ is contained in the $g_{3}^{1}$, and the line spanned by $p, q$ has contact order 3 at $p$ and 2 at $q$ to $Y$, hence $u$ must be $p$ or $q$ by Bézout. For $u=q$, subtracting $2 u$ from $3 p+2 q$, we know that $3 p$ is also in the $g_{3}^{1}$, hence $3 p \sim 2 p+q$, $p \sim q$, impossible. For $u=p$, we have $p+2 q$ is in the $g_{3}^{1}$. Hence $p+2 q \sim 2 p+q$, which implies $p \sim q$ and this is also impossible.

Proof of Theorem 1.3, Case $\Omega \mathcal{M}_{5}(5,3)$ Proposition 7.3 says that $\bar{C} \cdot B N_{4,(1,2)}^{1}=0$ for a Teichmüller curve $C$ in this stratum. Using the divisor class of $B N_{4,(1,2)}^{1}$ in $(10)$ together with Proposition 4.8, the result follows immediately. 


\subsection{The stratum $\Omega \mathcal{M}_{5}(6,2)^{\text {odd } *}$}

In the case $\Omega \mathcal{M}_{5}(6,2)^{\text {odd }}$ we have

$$
L_{(6,2)^{\text {odd }}}=46 / 21, \quad s_{(6,2)^{\text {odd }}}=176 / 23, \quad c_{(6,2)^{\text {odd }}}=209 / 144 .
$$

Based on numerical values on individual Teichmüller curves, we believe that the sum of Lyapunov exponents is nonvarying in this stratum. But we have not discovered a divisor to carry out the desired disjointness argument.

\subsection{Examples of square-tiled surfaces in $g=5$ and $g=6$}

In this section we list examples of square-tiled surfaces in $g=5$ to justify that the sum of Lyapunov exponents in the remaining strata is indeed varying.

In the stratum $\Omega \mathcal{M}_{5}(7,1)$ varying sum can be checked using the square-tiled surface

$$
\left(\pi_{r}=(12345678910), \pi_{u}=(1596)(24710)\right) .
$$

In the stratum $\Omega \mathcal{M}_{5}(5,2,1)$ varying sum can be checked using the square-tiled surface

$$
\left(\pi_{r}=(1234567891011), \pi_{u}=(111)(23)(46)(79)\right) .
$$

In the stratum $\Omega \mathcal{M}_{5}(5,1,1,1)$ varying sum can be checked using the square-tiled surface

$$
\left(\pi_{r}=(123456789101112), \pi_{u}=(112)(23)(46)(810)\right) .
$$

In the stratum $\Omega \mathcal{M}_{5}(4,4)^{\text {even }}$ varying sum can be checked using the square-tiled surface

$$
\left(\pi_{r}=(12345678910), \pi_{u}=(110)(29)(3568)\right) .
$$

In the stratum $\Omega \mathcal{M}_{5}(4,4)^{\text {odd }}$ varying sum can be cross-checked using, besides the hyperelliptic locus, the square-tiled surface

$$
\left(\pi_{r}=(12345678910), \pi_{u}=(110)(23)(56)(78)\right) .
$$

In the stratum $\Omega \mathcal{M}_{5}(4,3,1)$ varying sum can be checked using the square-tiled surface

$$
\left(\pi_{r}=(123456789101112), \pi_{u}=(11194)(210356)(712)\right) .
$$

In the stratum $\Omega \mathcal{M}_{5}(3,2,2,1)$ varying sum can be checked using the square-tiled surface

$$
\left(\pi_{r}=(123)(456789101112), \pi_{u}=(111)(10513)(27)\right) .
$$


In the stratum $\Omega \mathcal{M}_{5}(3,2,1,1,1)$ varying sum can be checked using the square-tiled surface

$$
\left(\pi_{r}=(123456789101112), \pi_{u}=(112)(24)(57)(810)\right) .
$$

In the stratum $\Omega \mathcal{M}_{5}(3,1,1,1,1,1)$ varying sum can be checked using the square-tiled surface

$$
\left(\pi_{r}=(12345678910111213), \pi_{u}=(114)(24)(68)(1012)\right) .
$$

To indicate that the phenomenon of nonvarying sum of Lyapunov exponents is restricted to low genus and special loci, such as eg the hyperelliptic locus, we show that already in $g=6$ the best candidates fail.

Proposition 7.4 For $g=6$ the sum of Lyapunov exponents is varying in the strata $\Omega \mathcal{M}_{6}(10)^{\text {odd }}$ and $\Omega \mathcal{M}_{6}(10)^{\text {even }}$.

Proof For $\Omega \mathcal{M}_{6}(10)^{\text {odd }}$ the sum for the measure supported on the whole stratum is $L_{(10)^{\text {odd }}}=\frac{82680540070}{35169130909}$ using [16], but the square-tiled surface

$$
\left(\pi_{r}=(1234567891011), \pi_{u}=(1357911)\right)
$$

provides an example with $L(C)=\frac{3166}{1375}$. In the even case $L_{(10)^{\text {even }}}=\frac{9085753953118}{3770001658049}$ but the square-tiled surface

$$
\left(\pi_{r}=(1234567891011), \pi_{u}=(157911)(24)\right)
$$

gives an example with $L(C)=\frac{244729}{101893}$.

\section{Hyperelliptic strata and moduli spaces of pointed curves}

Using Teichmüller curves in the hyperelliptic strata we reverse our engine to present an application for the geometry of moduli spaces of pointed curves.

In the study of the geometry of a moduli space, a central question is to ask about the extremality of a divisor class, eg if it has nonnegative intersection numbers with various curve classes on the moduli space. Now consider the moduli space $\overline{\mathcal{M}}_{g, 1}$ of genus $g$ curves with one marked point. Define a divisor class

$$
D_{1}=4 g(g-1) \omega_{\text {rel }}-12 \lambda+\delta,
$$

where $\delta$ is the total boundary class. Let $\mathcal{X} \rightarrow B$ be a complete one-dimensional family of stable one-pointed curves with smooth generic fibers. Harris [25, Theorem 1] showed that $D_{1} \cdot B$ is always nonnegative and asked further if this is optimal, ie if there exists 
such a family $B$ satisfying $D_{1} \cdot B=0$. The reader may also refer to [27, (6.31), (6.34)] for an expository explanation. Define another divisor class on the moduli space $\overline{\mathcal{M}}_{g, 2}$ of genus $g$ curves with two marked points:

$$
D_{2}=\left(g^{2}-1\right)\left(\psi_{1}+\psi_{2}\right)-12 \lambda+\delta,
$$

where $\psi_{i}$ is the first Chern class of the cotangent line bundle associated to the $i$-th marked point. By a completely analogous argument as in [25], one easily checks that $D_{2}$ has nonnegative intersection with any complete one-dimensional family of stable two-pointed curves with smooth generic fibers. Similarly one can ask if this is optimal. Below we show that in both cases the zero-intersection can be attained.

Theorem 8.1 Let $C_{1}, C_{2}$ be Teichmüller curves in generated by flat surfaces in $\Omega \mathcal{M}_{g}(2 g-2)^{\text {hyp }}$ and $\Omega \mathcal{M}_{g}(g-1, g-1)^{\text {hyp }}$, lifted to $\overline{\mathcal{M}}_{g, 1}$ and $\overline{\mathcal{M}}_{g, 2}$ using the zeros of Abelian differentials, respectively. Then we have

$$
\begin{aligned}
& \bar{C}_{1} \cdot D_{1}=0, \\
& \bar{C}_{2} \cdot D_{2}=0 .
\end{aligned}
$$

Proof By the value of $s\left(C_{1}\right)$ given in Corollary 4.3 and Proposition 4.8, we obtain

$$
\begin{gathered}
\frac{\bar{C}_{1} \cdot \lambda}{\bar{C}_{1} \cdot \omega_{\text {rel }}}=g^{2}, \\
\frac{\bar{C}_{1} \cdot \delta}{\bar{C}_{1} \cdot \omega_{\text {rel }}}=4 g(2 g+1) .
\end{gathered}
$$

Plugging them into the intersection $\bar{C}_{1} \cdot D_{1}$, an elementary calculation shows that $\bar{C}_{1} \cdot D_{1}=0$.

Similarly we have

$$
\begin{gathered}
\frac{\bar{C}_{2} \cdot \lambda}{\bar{C}_{2} \cdot\left(\psi_{1}+\psi_{2}\right)}=\frac{g(g+1)}{4}, \\
\frac{\bar{C}_{2} \cdot \delta}{\bar{C}_{2} \cdot\left(\psi_{1}+\psi_{2}\right)}=(g+1)(2 g+1) .
\end{gathered}
$$

One easily checks that $\bar{C}_{2} \cdot D_{2}=0$.

Since square-tiled surfaces in a stratum correspond to 'lattice points' under the period coordinates, the union of all such Teichmüller curves $C_{1}, C_{2}$ forms a Zariski dense subset in the hyperelliptic locus. Hence they provide infinitely many solutions to the above question. We finally remark that the positivity of $D_{1}$ as well as not being strictly 
ample has a transparent geometric explanation, pointed out to us by one of the referees. It is proportional to the pullback of the theta line bundle from the universal Jacobian over $\overline{\mathcal{M}}_{g, 1}$ via the map $(C, p) \mapsto\left[\mathcal{O}((2 g-2) p) \otimes \omega_{C}^{*}\right]$ by Morita [38, Theorem 1.6].

\section{References}

[1] E Arbarello, M Cornalba, The Picard groups of the moduli spaces of curves, Topology 26 (1987) 153-171 MR895568

[2] E Arbarello, M Cornalba, P A Griffiths, J Harris, Geometry of algebraic curves. Vol. I, Grundl. Math. Wissen. 267, Springer, New York (1985) MR770932

[3] M Bainbridge, Euler characteristics of Teichmüller curves in genus two, Geom. Topol. 11 (2007) 1887-2073 MR2350471

[4] A Beauville, Complex algebraic surfaces, second edition, London Mathematical Society Student Texts 34, Cambridge Univ. Press (1996) MR1406314

[5] I Bouw, M Möller, Teichmüller curves, triangle groups, and Lyapunov exponents, Ann. of Math. 172 (2010) 139-185

[6] L Caporaso, Linear series on semistable curves, Int. Math. Res. Not., rnq 188, 49 pages (2010)

[7] D Chen, Covers of elliptic curves and the moduli space of stable curves, J. reine angew. Math. 649 (2010) 167-205

[8] D Chen, Square-tiled surfaces and rigid curves on moduli spaces, Adv. Math. 228 (2011) 1135-1162

[9] D Chen, M Möller, Quadratic differentials in low genus: exceptional and non-varying strata arXiv:1204.1707

[10] M Cornalba, Moduli of curves and theta-characteristics, from: "Lectures on Riemann surfaces (Trieste, 1987)”, World Sci. Publ., Teaneck, NJ (1989) 560-589 MR1082361

[11] I Coskun, Degenerations of surface scrolls and the Gromov-Witten invariants of Grassmannians, J. Algebraic Geom. 15 (2006) 223-284 MR2199064

[12] F Cukierman, Families of Weierstrass points, Duke Math. J. 58 (1989) 317-346 MR1016424

[13] V Delecroix, P Hubert, S Leli'evre, Diffusion in the periodic wind-tree model arXiv: 1107.1810

[14] D Eisenbud, J Harris, The Kodaira dimension of the moduli space of curves of genus $\geq 23$, Invent. Math. 90 (1987) 359-387 MR0910206

[15] A Eskin, M Kontsevich, A Zorich, Sum of Lyapunov exponents of the Hodge bundle with respect to the Teichmüller geodesic flow arXiv: 1112.5872 
[16] A Eskin, H Masur, A Zorich, Moduli spaces of Abelian differentials: the principal boundary, counting problems, and the Siegel-Veech constants, Publ. Math. Inst. Hautes Études Sci. 97 (2003) 61-179 MR2010740

[17] A Eskin, A Okounkov, Asymptotics of numbers of branched coverings of a torus and volumes of moduli spaces of holomorphic differentials, Invent. Math. 145 (2001) 59-103 MR1839286

[18] G Farkas, Koszul divisors on moduli spaces of curves, Amer. J. Math. 131 (2009) 819-867 MR2530855

[19] G Farkas, The birational type of the moduli space of even spin curves, Adv. Math. 223 (2010) 433-443

[20] G Farkas, A Verra, The geometry of the moduli space of odd spin curves arXiv: 1004.0278

[21] H M Farkas, Unramified double coverings of hyperelliptic surfaces, J. Analyse Math. 30 (1976) 150-155 MR0437741

[22] G Forni, On the Lyapunov exponents of the Kontsevich-Zorich cocycle, from: "Handbook of dynamical systems. Vol. 1B”, Elsevier B. V., Amsterdam (2006) 549-580 MR2186248

[23] G Forni, C Matheus, An example of a Teichmüller disk in genus 4 with degenerate Kontsevich-Zorich spectrum arXiv:0810.0023

[24] J Harer, The second homology group of the mapping class group of an orientable surface, Invent. Math. 72 (1983) 221-239 MR0700769

[25] J Harris, Families of smooth curves, Duke Math. J. 51 (1984) 409-419 MR0747873

[26] J Harris, I Morrison, Slopes of effective divisors on the moduli space of stable curves, Invent. Math. 99 (1990) 321-355 MR1031904

[27] J Harris, I Morrison, Moduli of Curves, Graduate Texts in Mathematics 187, Springer, New York (1998) MR1631825

[28] J Harris, D Mumford, On the Kodaira dimension of the moduli space of curves, Invent. Math. 67 (1982) 23-88 MR664324 With an appendix by William Fulton

[29] B Hassett, Stable log surfaces and limits of quartic plane curves, Manuscripta Math. 100 (1999) 469-487 MR1734796

[30] K Kodaira, On compact analytic surfaces. II, III, Ann. of Math. 77 (1963), 563-626; ibid. 78 (1963) 1-40 MR0184257

[31] M Kontsevich, Lyapunov exponents and Hodge theory, from: "The mathematical beauty of physics (Saclay, 1996)”, Adv. Ser. Math. Phys. 24, World Sci. Publishing, River Edge, NJ (1997) 318-332 MR1490861

[32] M Kontsevich, A Zorich, Lyapunov exponents and Hodge theory arXiv: hep-th/9701164 
[33] M Kontsevich, A Zorich, Connected components of the moduli spaces of Abelian differentials with prescribed singularities, Invent. Math. 153 (2003) 631-678 MR2000471

[34] A Logan, The Kodaira dimension of moduli spaces of curves with marked points, Amer. J. Math. 125 (2003) 105-138 MR1953519

[35] H Masur, On a class of geodesics in Teichmüller space, Ann. of Math. 102 (1975) 205-221 MR0385173

[36] M Möller, Variations of Hodge structures of a Teichmüller curve, J. Amer. Math. Soc. 19 (2006) 327-344 MR2188128

[37] M Möller, Shimura and Teichmüller curves, J. Mod. Dyn. 5 (2011) 1-32 MR2787595

[38] S Morita, Families of Jacobian manifolds and characteristic classes of surface bundles, II, Math. Proc. Cambridge Philos. Soc. 105 (1989) 79-101 MR0966142

[39] D Mumford, Stability of projective varieties, Enseignement Math. 23 (1977) 39-110 MR0450272

[40] M Reid, Chapters on algebraic surfaces, from: "Complex algebraic geometry (Park City, UT, 1993)”, IAS/Park City Math. Ser. 3, Amer. Math. Soc., Providence, RI (1997) 3-159 MR1442522

[41] F Yu, K Zuo, Weierstrass filtration on Teichmüller curves and Lyaponov exponents arXiv:1203.6053

[42] A Zorich, Flat surfaces, from: "Frontiers in Number Theory, Physics and Geometry. Volume 1: On random matrices, zeta functions and dynamical systems", Springer, Berlin (2006) 439-586

Department of Mathematics, Boston College

Carney Hall, 140 Commonwealth Avenue, Chestnut Hill, MA 02467, USA

Institut für Mathematik, Goethe-Universität Frankfurt

Robert-Mayer-Str. 6-8, D-60325 Frankfurt am Main, Germany

dawei.chen@bc.edu, moeller@math.uni-frankfurt.de

Proposed: Benson Farb

Seconded: Shigeyuki Morita, Walter Neumann

Received: 19 August 2011

Revised: 30 July 2012 